\title{
miR825 and miR825* function as negative regulators in Bacillus cereus AR156-elicited systemic resistance to Botrytis cinerea in Arabidopsis thaliana
}

\author{
Pingping Nie \\ Nanjing Agricultural University \\ Chen Chen \\ Nanjing Agricultural University \\ Qian Yin \\ Jiangsu Province and Chinese Academy of Science \\ Chunhao Jiang \\ Nanjing Agricultural University \\ Jianhua Guo \\ Nanjing Agricultural University \\ Hongwei Zhao \\ Nanjing Agricultural University \\ Dongdong Niu ( $\nabla$ ddniu@njau.edu.cn ) \\ Nanjing Agricultural University College of Plant Protection
}

Research article

Keywords: miR825 and miR825*, Bacillus cereus AR156, Induced systemic resistance, Botrytis cinerea B1301, Plant innate immunity

Posted Date: July 5th, 2019

DOI: https://doi.org/10.21203/rs.2.10988/v1

License: (c) (i) This work is licensed under a Creative Commons Attribution 4.0 International License.

Read Full License

Version of Record: A version of this preprint was published at International Journal of Molecular Sciences on October 11th, 2019. See the published version at https://doi.org/10.3390/ijms20205032. 


\section{Abstract}

Background: Small RNAs function to regulate plant defense responses to pathogens. We previously showed that miR825 and miR825* downregulate Bacillus cereus AR156 (AR156)-triggered systemic resistance to Pseudomonas syringae pv. tomato DC3000 in Arabidopsis thaliana (Arabidopsis). The aim of this study was to unravel the role of miR825 and miR825* in AR156-mediated systemic resistance to Botrytis cinerea B1301 in Arabidopsis. Results: Northern blotting revealed that miR825 and miR825* were more strongly downregulated in wild type Arabidopsis Col-0 (Col-0) plants pretreated with AR156 than in non-treated plants upon B. cinerea B1301 infection. Furthermore, compared with Col-0, transgenic plants with attenuated miR825 and miR825* expression were more resistant to B. cinerea B1301, yet miR825and miR825*-overexpressing (OE) plants were more prone to it. With AR156 pretreatment, the transcription of four defense-related genes (PR1, PR2, PR5, and PDF1.2) and cellular defense responses (hydrogen peroxide production and callose deposition) were faster and stronger in miR825 and miR825* knockdown lines, but weaker in their OE plants than in Col-0 plants upon pathogen attack. Also, AR156 pretreatment caused stronger phosphorylation of MPK3 and MPK6 and expression of FRK1 and WRKY53 genes upon B. cinerea B1301 inoculation in miR825 and miR825* knockdown plants than in Col-0 plants. Additionally, the assay of agrobacterium-mediated transient co-expression in Nicotiana benthamiana confirmed that AT5G40910, AT5G38850, AT3G04220, and AT5G44940 are target genes of miR825 or miR825*. Compared with Col-0, the target mutant lines showed higher susceptibility to B. cinerea B1301 while still expressing AR156-triggered ISR. The two-way ANOVA revealed a significant $(P<0.01)$ interactive effect of treatment and genotype on the defence responses. Conclusion: miR825 and miR825* act as negative regulators of AR156-mediated systemic resistance to B. cinerea B1301 in Arabidopsis. Our research have significant implications for effectively applying the two miRNAs to plant protection.

\section{Background}

Plants are equipped with sophisticated immune response mechanisms to resist pathogen attack [1, 2]. Pattern-recognition receptors (PRRs) constitute the first line of plant defence against pathogens by recognizing conserved pathogen-associated molecular patterns (PAMPs), resulting in PAMP-triggered immunity (PTI). The components of PTI include mitogen-activated protein kinase (MAPK) activation [3], defense-related gene expression, and callose deposition $[2,4,5]$. On the other hand, many pathogens secret multiple specific effectors to inhibit PTI in host plants [6-8], which, however, have developed the second line of defense comprising resistance $(R)$ proteins that target corresponding pathogen effectors, resulting in effector-triggered immunity (ETI) [9] hypersensitive response (HR) at the infected site to inhibit the growth of biotrophic pathogens $[1,2]$.

Induced disease resistance in plants is effective in controlling infections of a wide variety of pathogens (bacteria, fungi, and viruses), as well as insect herbivores [10-12]. Systemic acquired resistance (SAR) and induced systemic resistance (ISR) are two types of well-studied induced resistance [13], both resulting in defense responses in local and systemic tissues $[10,14,15]$. Usually, plants express SAR when infected with a necrotizing pathogen [14], while ISR is triggered by some beneficial rhizobacteria including plant- 
growth-promoting rhizobacteria (PGPR), such as Pseudomonas and Bacillus [10, 16, 17]. A large number of plant species have been found to express ISR, including tomato, rice, tobacco, cucumber, bean, and the model plant Arabidopsis thaliana. By expressing ISR, plants can effectively resist the attack of pathogenic microorganisms (bacteria, fungi, and viruses), as well as insect herbivores $[12,17,18]$. Rhizobacteria elicit ISR through priming; upon pathogen attack, plants at the primed state mount fast and strong cellular defense responses comprising cell-wall reinforcement [19], oxidative burst [20], expression of defense-related enzymes [21], and accumulation of secondary metabolites [22].

The phytohormones salicylic acid (SA), jasmonic acid (JA), and ethylene (ET) play essential roles in expressing SAR and/or ISR [23]. In SAR-expressing plants, levels of endogenously synthesized SA increase systemically, resulting in enhanced expression of the genes that encode pathogenesis-related (PR) proteins, such as PR1, PR2, and PR5 [10, 24, 25]. Differently, ISR is activated through the JA/ETdependent signaling pathway, involving plant defensin 1.2 (PDF1.2) $[18,26]$. For instance, the ISR induced by Pseudomonas fluorescens WCS417r in Arabidopsis is dependent on the JA/ET signaling pathway and NPR1 [27], as is the ISR elicited by Bacillus cereus AR156 (AR156) against Botrytis cinerea (B. cinerea) B1301 in Arabidopsis [28]. However, some rhizobacteria including PGPR were shown to trigger ISR through both SA- and JA/ET- dependent signaling pathways [10, 29].

Small RNAs (sRNAs) function to mediate plant defense responses against pathogens [8, 30, 31-33]. sRNAs consist of small interfering RNAs (siRNAs) and microRNAs (miRNAs), which vary in biogenesis and precursor structure [8]; they can bind argonaute (AGO) proteins, forming RNA-mediated silencing complex to regulate gene expression. miR393 is the first example identified as PTI-related SRNA; its expression is elicited by flg22, a well-studied PAMP molecule, triggering PTI by inhibiting auxin signaling via silencing its receptors [34]. Moreover, miR773, miR160a, and miR398b act to regulate the deposition of callose, therefore participating in PTI [35]. On the other side, some miRNAs are involved in ETI signaling; for example, miR393b ${ }^{*}$ targets a Golgi-localized SNARE gene, which stimulates exocytosis of an antimicrobial pathogenesis-related protein, thus regulating plant defense [36]. miRNAs also regulate the expression of defense-related host resistance $(R)$ genes [37, 38]. For instance, miR1885, whose expression is activated by Turnip mosaic virus (TuMV) infection in Brassica, targets the TIR-NBS-LRR class $R$ genes [39]; miR482, whose expression is suppressed by virus infection, targets the NBS-LRR class $R$ genes and therefore suppresses tomato defense against pathogen attack [40]. In addition, enhanced expression of miR6019 and miR6020 leads to downregulation of $R$ genes, causing attenuation of $R$ genes-dependent defense responses to tobacco mosaic virus (TMV) in Nicotiana benthamiana [38]. Besides, miR472 downregulates PTI, as well as the ETI triggered by resistance Pseudomonas syringae 5 (RPS5) by targeting coiled-coil (CC)-NBS-LRR genes in Arabidopsis [41].

We previously reported that AR156 triggers ISR to Pseudomonas syringae pv. tomato (Pst) DC3000 and B. cinerea B1301 in Arabidopsis [10, 28]. Moreover, we found that miR825 and miR825 in Arabidopsis act as negative regulators of AR156-mediated ISR to Pst DC3000 by repressing the expression of defenserelated genes [42]. On the basis of these findings, the present study was undertaken to elucidate the function of miR825 and miR825* in AR156-mediated ISR against B. cinerea B1301. As a result, northern 
blotting revealed that upon challenge inoculation with $B$. cinerea B1301, stronger downregulation of miR825 and miR825* expression occurred in AR156-pretreated plants than in non-treated control plants. On the other hand, miR825- and miR825 -overexpressing (OE) plants showed a higher susceptibility to $B$. cinerea B1301 than Col-0; in contrast, the short tandem target mimic (STTM) miR825 and miR825* (STTM825/825*) transgenic lines were more resistant to it. Moreover, upon B. cinerea B1301 infection, cellular defense responses (hydrogen peroxide production and callose deposition) and expression of defense-related genes were stronger in AR156-pretreated plants from miR825/825 knockdown lines, but weaker in those from miR825 and miR825* OE plants than in Col-0 plants. We also identified a number of $R$ genes of the TIR-NBS-LRR class as miR825* targets, which were expressed in a similar manner during AR156-triggered ISR. Furthermore, the target mutant plants were more prone to $B$. cinerea B1301 infection than Col-0; on the other side, AR156 still induced an effective ISR in target mutant lines. All in all, this study indicated that miR825 and miR825 function to inhibit AR156-elicited ISR to $B$. cinerea by repressing defense-related gene expression and cellular defense responses.

\section{Methods}

\section{Plant materials and growth conditions}

Plants of Arabidopsis thaliana and Nicotiana benthamiana were cultivated in a growth chamber maintained at $23 \pm 1^{\circ} \mathrm{C}$ in a photoperiod of 12-h light/12-h dark. Arabidopsis lines used in this study were as follows: Col-0 (Arabidopsis thaliana ecotype); two transgenic plant lines (\#50 and \#56), each overexpressing both miR825 and miR825* two STTM825/825* transgenic plant lines (\#1 and \#3) [42]; and the T-DNA insertion mutants at5g44940 (SALK_021558C), at5g38850 (SALK_134889C), at5g40910 (SALK_043422C), and at3g04220 (CS384498), and the seeds of Col-0 and Nicotiana benthamiana were kindly provided by Hansong Dong (Nanjing Agricultural University), Dongdong Niu constructed and identified overexpressing and STTM of miR825/825* transgenic plant lines, and the seeds of four T-DNA insertion mutants were obtained from the College of Arts and Sciences Arabidopsis Biological Resource Center (ABRC, The Ohio State University, US). Homozygous mutant plants were identified by using semiquantitative RT-PCR, and the primers are shown in Additional file 4: Table S1. Three-week-old plants were used in all experiments.

\section{Induction treatments and pathogen infection}

The tested PGPR strain B. cereus AR156 was cultured on Luria-Bertani (LB) agar plates, which were incubated at $28^{\circ} \mathrm{C}$ for $24 \mathrm{~h}$. Bacteria were subsequently collected by centrifugation, followed by their resuspension in a sterile $0.85 \% \mathrm{NaCl}$ solution with a final concentration of $5 \times 10^{8} \mathrm{CFU} / \mathrm{ml}$. To prepare the inoculum of $B$. cinerea B1301, the strain was cultured on potato sucrose agar (PSA) for 7 days; its spores were collected in $\mathrm{H}_{2} \mathrm{O}$, from which a spore suspension with a final concentration of $1 \times 10^{6}$ spores $/ \mathrm{ml}$ was 
prepared. Each plant was applied with $7 \mathrm{ml}$ of the AR156 cell suspension or a sterile $0.85 \% \mathrm{NaCl}$ solution (the control) as a root drench; 7 days later, plants were challenge inoculated by dropping a $10 \mu$ droplet of the spore suspension of $B$. cinerea B1301 on the midvein of each side of leaves. Then all plants were grown in a growth chamber maintained at $23 \pm 1^{\circ} \mathrm{C}$ and with $70 \%$ relative humidity for 2 days. Transcript levels of $B$. cinerea actin gene (BcActin) were measured for determining in planta growth rate of $B$. cinerea B1301, employing the Arabidopsis actin 1 gene (AtActin1) as an internal control. The ratio of BcActin to AtActin 1 (BcActin/AtActin 1) was calculated to indicate relative fungal growth rate.

For Phytophthora capsici (P. capsici) infection of Arabidopsis, P. capsici was cultured as reported by Lu [59]. The number of $P$. capsici zoospores in $10 \mu \mathrm{l}$ water was counted under a microscope to determine its concentration. Leaves were detached from plants of Col-0, miR825/825* OE lines (\#50 and \#56), and STTM825/825* lines (\#1 and \#3); and then each leaf was put on a piece of wet paper in a petri dish. Approximately $100 \mathrm{P}$. capsici zoospores were dropped onto the center of each leaf, which was then incubated in a growth room maintained at $25^{\circ} \mathrm{C}$ in darkness.

For virus inoculations, transient expression assays in $N$. benthamiana were conducted by syringeinfiltrating leaves of three-week-old Nicotiana benthamiana plants with a cell suspension of Agrobacterium $\left(\mathrm{OD}_{600}=1.0\right)$ harbouring constructs containing cucumber mosaic virus $(\mathrm{CMV}) . N$. benthamiana leaves were sampled at 7 days post inoculation (dpi); ground in $1 \times \mathrm{PBS}$; and then rubinoculated onto leaves of plants from Col-0, miR825/825* OE lines (\#50 and \#56), and STTM825/825* lines (\#1 and \#3). All virus-inoculated plants were grown in growth chambers (model GXZ500D, Jiangnan Motor Factory, Ningbo, China) maintained at $25^{\circ} \mathrm{C}$ with $8 \mathrm{~h}$ light/ $16 \mathrm{~h}$ dark.

\section{RNA extraction, quantitative RT-PCR, and RNA gel blot analysis}

Total RNA in Arabidopsis leaves was extracted with TRIzol Reagent (Invitrogen, San Diego, CA, U.S.A). Briefly, cDNA was synthesized by using $1 \mu \mathrm{g}$ total RNA with a commercial reverse transcription system (TaKaRa Biotech, Dalian, China). Real-time quantitative RT-PCR was performed by using $2 \mu$ cDNA that was $10 x$ dilution of the synthesis product, under the following reaction conditions: 40 cycles of denaturing at $95^{\circ} \mathrm{C}$ for $30 \mathrm{~s}$, annealing at $55^{\circ} \mathrm{C}$ for $30 \mathrm{~s}$, and extension at $72^{\circ} \mathrm{C}$ for $34 \mathrm{~s}$. Each sample comprised three replications. The data were normalized with AtActin 1.

RNA gel blot analysis was conducted using the method of Yan [60]. RNA samples were resolved on a denaturing $17 \%$ polyacrylamide gel; subsequently, the RNA molecules separated by the electrophoresis were transferred to Hybond- $\mathrm{N}^{+}$membranes electrophoretically (Amersham BioScience). DNA oligonucleotide probes, ${ }^{32} \mathrm{P}$-labeled by using T4 polynucleotide kinase (New England Biolabs), were utilized in hybridization reactions conducted at $38^{\circ} \mathrm{C}$ in PerfectHyb Plus buffer. A phosphorimager was employed to detect hybridization signals. The expression of the miRNAs was quantified, and the resultant values were normalized with $\mathrm{U} 6$, resulting in the relative abundance (RA) of miRNAs. The sequences of 
primers used in the quantitative RT-PCR and northern blotting assays are listed in Additional file 4: Table S1.

\section{Detection of hydrogen peroxide accumulation and callose deposition}

Three-week-old Arabidopsis plants from Col-0, miR825/825* OE lines (\#50 and \#56) and STTM825/825* lines (\#1 and \#3) were pretreated by applying $7 \mathrm{ml}$ of the AR156 suspension at $5 \times 10^{8} \mathrm{CFU} / \mathrm{ml}$ or a sterile $0.85 \% \mathrm{NaCl}$ solution (the control) to each plant as a root drench. At $7 \mathrm{dpt}$, B. cinerea B1301 spores were collected to make a spore suspension with the final concentration at $1 \times 10^{6} \mathrm{spores} / \mathrm{ml}$. Plants were challenge inoculated by dropping a $10 \mu \mathrm{l}$ droplet of the spore suspension on the midvein of each side of a leaf. At 12 and 24 hours post inoculation (hpi), Arabidopsis leaves were sampled and then processed for detecting the accumulation of hydrogen peroxide and the deposition of callose using the protocals described in our earlier study (Niu et al., 2011). In brief, for ROS accumulation, leaves of each sample were collected from at least three different plants, which were stained with a diaminobenzidine (DAB) solution $(1 \mathrm{mg} / \mathrm{mL}, \mathrm{pH} 3.8)$ for $8 \mathrm{~h}$ in the darkness at $25-28^{\circ} \mathrm{C}$. Subsequently, $96 \%$ (vol/vol) ethanol was used to clear the stained leaves, which were finally preserved in $50 \%$ (vol/vol) ethanol. Under the light microscope, hydrogen peroxide was visualized as dark-brown precipitate in a DAB-stained leaf.

To detect the deposition of callose, leaves were soaked in $5 \mathrm{ml}$ of a destaining solution consisting of acetic acid and ethanol in a ratio of 5:95 (vol/vol), followed by vacuum infiltration for 5-10 min. Leaf chlorophyll was cleared by incubating leaves in a $60^{\circ} \mathrm{C}$ water bath for 20-30 min. After being gently rinsed with water, the resultant chlorophyll-free leaves were immersed in $3-5 \mathrm{ml}$ of a staining solution that contained $0.01 \%(\mathrm{wt} / \mathrm{vol})$ aniline blue and $150 \mathrm{mM} \mathrm{K}_{2} \mathrm{HPO}_{4}(\mathrm{pH} 9.5)$ in the darkness for $2-4 \mathrm{~h}$. After being gently rinsed with water, the stained leaves were mounted on microscope slides for observation under an epifluorescence microscope equipped with a UV excitation filter.

\section{Protein extraction and Western blotting analysis}

Three-week-old plants of Arabidopsis Col-0, miR825/825* OE transgenic line \#50, and STTM825/825 transgenic line \#1 were applied with AR156 or $0.85 \% \mathrm{NaCl}$ (the control) as described above. At $7 \mathrm{dpt}$, challenge inoculation was carried out. Leaves were sampled at 0, 10, 30, and 60 mpi. Subsequently, they were ground in liquid nitrogen, followed by extraction of total proteins with a $2 \times$ SDS loading buffer. The extracted proteins were separated on $12 \%$ SDS-PAGE gels, and then the separated protein molecules were transferred onto nitrocellulose membranes, followed by blotting with a monoclonal mouse anti-HA (1:5,000 dilution) and a monoclonal mouse anti-a tubulin (1:5,000 dilution). To measure MAPK activity, samples were subjected to Western blotting with monoclonal rabbit phospho-p44/42 MAPK (Erk1/2) (Thr202/Tyr204) (D13.14.4E) XP antibodies (Cell Signaling Technology, \#4370S, 1:2,000 dilution), and atubulin was included as a loading control. 


\section{Transient expression analysis in Nicotiana benthamiana}

To generate the expression constructs of miR825, miR825* and miR319b, the primary constructs amiR825, amiR825* and amiR319b were engineered from pRS300 (miR825, miR825* and miR319b) Arabidopsis thaliana by using the WMD3 online tool (http: //wmd3.weigelworld.org), which were subsequently cloned into a pEarleyGate (pEG202) destination vector by LR clonase II (Invitrogen). The corresponding miRNA overexpression plant lines were generated by cloning the coding sequences of At5G40910, At5G38850, At3G04220, and At5G44940 into the pEG201 vector.

In transient co-expression and co-immunoprecipitation assays, leaves of three-week-old $N$. benthamiana plants were syringe-infiltrated with a cell suspension of Agrobacterium tumefaciens GV3101 $\left(\mathrm{OD}_{600}=\right.$ 1.0) harbouring constructs containing miR825 (pEG202) and miR825* (pEG202), as well as that of $A$. tumefaciens $\mathrm{GV} 3101\left(\mathrm{OD}_{600}=1.0\right)$ containing the coding sequences of the four target genes ( $\left.p E G 201\right)$, while miR319b ( $p E G 202$ ) was employed as the control. Leaf tissues were collected at $48 \mathrm{hpi}$ and processed as described above, and protein expression was detected by Western blotting.

\section{Statistical analysis}

Two-way ANOVA analysis with Tukey's test was conducted to detect the interaction between two independent variables: treatment (AR156 or Control) and plant genotype. The Student's $t$-test was carried out to assess statistical significance for all pairwise comparisons. Single and double asterisks above the columns in figures indicate significant $\left({ }^{*} P<0.05\right)$ and very significant $(* * P<0.01)$ differences, respectively, between AR156 and Control treatments in plants with different genotypes, as well as those between different genotypes. Standard deviations were calculated. All analyses were conducted with SPSS software for Windows version 19.0 (IBM Co., Armonk, NY, USA).

\section{Results}

\section{miR825 and miR825 ${ }^{*}$ expression was suppressed in AR156-induced ISR to B. cinerea in Arabidopsis.}

To decipher the function of miR825 and miR825 ${ }^{*}$ in AR156-triggered ISR to B. cinerea B1301 in Arabidopsis, wild-type Col-0 plants were pretreated with AR156 or $0.85 \% \mathrm{NaCl}$ as a root drench and then inoculated with B. cinerea B1301 or water (mock) at 7 days post-treatment (dpt). As shown in Fig. 1a, at 2 days post-inoculation (dpi), non-treated control plants (Control/Mock) and AR156-treated plants (AR156/Mock), both of which were mock-inoculated, showed no symptoms; by contrast, with $B$. cinerea B1301 inoculation, both of non-treated control plants (Control/B. cinerea) and AR156-treated plants (AR156/B. cinerea) developed typical symptoms of gray mould disease (severe necrotic lesions surrounding inoculating loci, leaf yellowing, and watersoaked spots covered with B. cinerea B1301 
spores), while the symptoms were less pronounced in AR156/B. cinerea than in Control/ B. cinerea, indicating that AR156 pretreatment enhanced plant resistance to $B$. cinerea B1301. To investigate whether miR825 and miR825 ${ }^{*}$ have function in the mediation of AR156-triggered ISR against $B$. cinerea B1301, we reasoned that if having this function, they may be differentially expressed during the process of priming or upon $B$. cinerea B1301 inoculation. Therefore, we examined the expression of the two miRNAs in AR156/B. cinerea, Control/B. cinerea, AR156/Mock, and Control/Mock at 48 hours postinoculation (hpi). Northern blotting analysis confirmed that miR825* expression was downregulated with B. cinerea B1301 infection in both AR156-treated and control plants. On the one hand, there was no appreciable difference in the level of miR825* expression between AR156/Mock and Control/Mock, yet its level was significantly lower in AR156/B. cinerea than in Control/B. cinerea, and the difference in the same parameter between Control/Mock and Control/B. cinerea was smaller than that between AR156/Mock and AR156/B. cinerea (Fig. 1b). In addition, we also investigated the role of miR825 in ISR. On the one hand, no difference in miR825 expression level was noted between AR156/Mock and Control/Mock, which was similar to the expression profile of miR825; on the other hand, though miR825 expression was also downregulated upon $B$. cinerea B1301 infection in control plants, the downregulation was significantly stronger in AR156-treated plants (Fig. 1b). The results indicated that AR156 pretreatment resulted in significant suppression of the expression of miR825 and miR825*, suggesting that they might function as negative regulators of AR156-mediated ISR to B. cinerea B1301.

\section{miR825 and miR825* participate in AR156-induced ISR.}

To further investigate whether miR825 and miR825 ${ }^{*}$ function in AR156-elicited ISR to B. cinerea B1301, the ability of AR156 to trigger ISR was evaluated in the transgenic plants in which miR825 and miR825 were knockdowned by using STTM strategy and in miR825 and miR825* OE plants. Two-way ANOVA with Tukey's test was used to compare the levels of resistance to B. cinerea B1301 in AR156-treated and non-treated plants with different genotypes. Typical symptoms of gray mould disease (severe necrosis and watersoaked spots surrounded by the spores) appeared on leaves of Control/ $B$. cinerea from Col- 0 at 2 dpi (Fig. 2a); in comparison, disease symptoms were more severe in those from miR825/825 ${ }^{*}$ OE lines (\#50 and \#56), which formed larger necrotic lesions, but lighter in those from STTM825/825* transgenic lines (\#1 and \#3) (Fig. 2a). However, AR156 pretreatment led to marked reductions in disease symptoms regardless of plant lines (Fig. 2a). On the other side, compared with Col-0, STTM825/825* lines (\#1 and \#3) expressed stronger resistance to Phytophthora capsici (P. capsici) (Additional file 1: Figure S1A) and cucumber mosaic virus (CMV) (Additional file 1: Figure S1B), and showed significant ( $* \star P<0.01$ ) reductions in the lesion diameter on the leaf infected by $P$. capsici (Additional file 1: Figure S1C) and in that by CMV (Additional file 1: Figure S1D), indicating that miR825 and miR825 ${ }^{*}$ act as negative regulators of Arabidopsis resistance to various pathogens (B. cinerea, P. capsici, and CMV). Compared to Col-0 plants, the diameter of leaf necrosis was smaller in the two STTM825/825* lines, but bigger in the two miR825/825* OE lines, and AR156-treated plants from STTM825/825* lines developed significantly 
$(\star \star P<0.01)$ smaller necrotic lesions on leaves than the corresponding control plants (Fig. 2b).

Additionally, qRT-PCR was conducted to measure B. cinerea B1301 growth rate at $2 \mathrm{dpi}$ in infected leaves. Coincident with the reduction in disease symptoms on AR156-treated leaves, the growth rate of $B$. cinerea B1301 was consistently lower in leaf tissues of AR156-pretreated plants with three different genotypes than in respective control plants; with AR156 pretreatment, the fungal growth rate was lower in the STTM825/825 lines, but higher in the miR825/825 ${ }^{*}$ OE lines than in Col-0 plants (Fig. 2c). The two-way ANOVA revealed that the interaction between treatment (AR156, Control) and genotype was statistically significant and that AR156-pretreated plants with three different genotypes overall showed significantly higher levels of resistance to $B$. cinerea B1301 than respective control plants. Collectively, these results implicate that miR825 and miR825 ${ }^{\star}$ function to suppress innate immunity in Arabidopsis that protect plants from the infection of a wide variety of pathogens (B. cinerea, P. capsici, and CMV).

\section{miR825 and miR825* affect defense-related gene expression and cellular defense responses in AR156- primed plants upon pathogen attack.}

To identify the signaling pathway(s) involved in AR156-induced ISR, qRT-PCR was used to examine expression levels of the reporter genes of the SA-signaling pathway $(P R 1, P R 2$, and $P R 5)$ and that of the JA/ET-signaling pathway (PDF1.2). The expression of these genes in plants with different genotypes was analysed with two-way ANOVA. Without B. cinerea B1301 infection, their expression levels in the miR825/825* OE lines (\#50 and \#56) and STTM825/825* transgenic lines (\#1 and \#3) were comparable to those in the wild-type plants at $48 \mathrm{hpi}$ (Fig. 3a). However, their transcription was significantly enhanced in AR156/B. cinerea relative to that in Control/B. cinerea at 48 hpi regardless of plant lines (Fig. 3a). Moreover, the two-way ANOVA revealed that the interaction between treatment effect and genotype effect was statistically significant; with AR156 pretreatment, the transcriptional levels of these genes significantly increased in the STTM $825 / 825^{*}$ transgenic plants, but declined in the miR825/825* OE plants compared with those in Col-0 plants (Fig. 3a), which conformed to the function of miR825/825* as negative regulators of AR156-mediated ISR to $B$. cinerea.

We also investigated whether miR825 and miR825 ${ }^{\star}$ act in AR156 priming the plant for potentiated cellular defense responses, represented by the accumulation of hydrogen peroxide and the deposition of callose, in Arabidopsis upon pathogen attack. As shown in Fig. 3b, the two events were evident at $12 \mathrm{hpi}$ in AR156/B. cinerea of both Col-0 and STTM825/825 transgenic plants (lines 1 and 3), but much weaker in that of miR825/825* OE plants (lines 50 and 56); meanwhile, they were absent in Control/ B. cinerea. With AR156 pretreatment, these defense responses were potentiated in Col-0 and the two STTM825/825* transgenic lines at $24 \mathrm{hpi}$; meanwhile, they were weaker in both Control/B. cinerea and AR156/B. cinerea of miR825/825* OE plants (Fig. 3b). The observed results indicated that upon pathogen attack, AR156treated Arabidopsis plants expressed faster and stronger cellular defense responses, which, however, could be dampened by miR825 and miR825*. 


\section{miR825 and miR825* participate in regulation of PTI components in AR156-elicited ISR.}

With the purpose of elucidating the role of miR825 and miR825* in AR156-triggered ISR and PTI, the phosphorylation of MPK3 and MPK6 was examined in both control and AR156-pretreated plants inoculated with $B$. cinerea B1301. Using antibodies specifically recognizing MPK3 and MPK6, their phosphorylation was detected in leaves of Control/B. cinerea of Col-0 at 10 min post-inoculation (mpi); then its intensity gradually declined at 30 and $60 \mathrm{mpi}$. However, AR156 pretreatment caused sustained phosphorylation of the two kinases in leaves of Col-0 plants; its intensity increased with time, peaking at $60 \mathrm{mpi}$ (Fig. 4a). In Control/B. cinerea and AR156/B. cinerea from miR825/825* OE line (\#50), the tendency of MPK3 and MPK6 phosphorylation was the same as that in the corresponding treatments from Col-0 (Fig. 4b). Notably, the phosphorylation of the two kinases was sustained from 10 to $60 \mathrm{mpi}$ in both Control/ B. cinerea and AR156/B. cinerea of miR825/825 ${ }^{*}$ knockdown line (\#1), with its intensity remaining substantially higher in AR156/B. cinerea than in Control/B. cinerea over the time course (Fig. 4c). These results suggested that AR156-triggered ISR involves an enhanced and sustained PTI response, in which miR825 and miR825* play a negative regulatory role.

To search further evidence to support our hypothesis, we evaluated the transcription levels of two PTI marker genes: the flg22-induced receptor-like kinase 1 (FRK1) and WRKY53 [43, 44]. Differences in their expression between AR156-pretreated and control plants with different genotypes upon B. cinerea B1301 infection were analysed by two-way ANOVA. The qRT-PCR assay revealed that the expression of FRK1 and WRKY53 was enhanced and sustained in control/B. cinerea of Col-0, miR825/825 $5^{*}$ E line (\#50), and STTM825/825 ${ }^{\star}$ transgenic line (\#1) from 10 to $60 \mathrm{mpi}$. However, their expression significantly increased at 30 and $60 \mathrm{mpi}$ in the AR156/B. cinerea of STTM825/825* lines relative to that in the corresponding control plants (Fig. 4d). The two-way ANOVA revealed a significant $(P<0.01)$ interactive effect of treatment and genotype. These results confirmed that miR825 and miR825* negatively regulate the expression of PTI components in AR156-mediated ISR.

\section{Target genes of miR825 and miR825* are expressed in a similar manner in AR156-induced ISR.}

To investigate whether miR825 and miR825* modulate ISR by affecting the expression of their targets genes, qRT-PCR was conducted to examine expression patterns of miR825/825* target genes in control and AR156-pretreated plants of Col-0, miR825/825* OE lines (\#50 and \#56), and STTM825/825* lines (\#1 and \#3) with or without $B$. cinerea B1301 inoculation. We predicted that miR825/825* targets and their cognate miRNAs would exhibit opposite expression patterns in ISR-expressing plants. The expression of the targeted genes in plants with different genotypes treated with or without AR156 was analyzed using two-way ANOVA. As expected, the results of qRT-PCR indicated that the transcriptional levels of three miR825*-targeted genes (AT5G40910, AT5G38850, and AT3G04220) and a miR825-targeted gene 
(AT5G44940) were all significantly improved in leaves of both control and AR156-pretreated plants from miR825/825* knockdown lines (\#1 and \#3) with or without B. cinerea B1301 inoculation compared with those from Col-0 at $48 \mathrm{hpi}$. In contrast, their expression levels were consistently reduced in four treatments (Control/Mock, AR156/Mock, Control/B. cinerea, and AR156/B. cinerea) from miR825/825* OE lines (\#50 and \#56) compared with Col-0 at the same time. Moreover, AR156-induced transcription of the four target genes was significantly stimulated in the STTM825/825 $5^{\star}$ transgenic plants, but attenuated in the miR825/825* OE plants relative to that in Col-0 plants (Fig. 5a). At the same time, AR156 pretreatment coupled with $B$. cinerea B1301 infection led to conspicuous up- and down-regulation of these genes in STTM825/825* and miR825/825* OE plants, respectively, compared with that in Col-0 plants (Fig. 5a). Two-way ANOVA revealed that the interaction between treatment effect and genotype effect was statistically significant. Hence, it is clear that miR825 and mi825 ${ }^{\star}$ function to suppress AR156elicited ISR.

To further confirm that these genes are targeted by miR825 and miR825*, the two miRNAs were separately transiently co-expressed with a target gene fused with the HA-tag in Nicotiana benthamiana. An anti-HA antibody was used to detect the proteins encoded by AT5G40910, AT5G38850, AT3G04220, and $A T 5 G 44940$. As a result, their expression was apparently repressed by the co-expression of miR825/825, but not by miR319b, which is irrelevant to these genes (Fig. $5 b$ ). Taken together, these results signified that the four genes are authentic miR825- or miR825 ${ }^{*}$-targeted genes participating in AR156-induced ISR against B. cinerea B1301.

\section{Plants silencing miR825/miR825*targeted genes are more susceptible to $B$. cinerea.}

To clarify the function of the target genes of miR825 and miR825 ${ }^{*}$, they were evaluated in a functional analysis by using the null mutants at5g40910 (SALK_043422C), at5g38850 (SALK_134889C), at3g04220 (CS384498), and at5g44940 (SALK_021558C), each with a T-DNA insertion in an intron or exon (Additional file 2: Figure S2A). Semi-quantitative RT-PCR confirmed that the two mutants At5g38850 and At3g04220 were homozygous mutants (Additional file 2: Figure S2B); however, this assay also indicated that we failed to obtain the homozygous mutants of AT5G40910 and AT5G44940 (Additional file 3: Figure S3), and we speculate that this failure might be caused by homozygous infertility or T-DNA insertion mutation. The germination rate of seeds was similar among Col-0, at5g38850, and at3g04220 (Additional file 2: Figure S2C). The mutants at5g38850 and at3g04220 phenotypically developed to a lesser degree than Col-0 plants (Additional file 2: Figure S2D). To elucidate the role of these target genes in AR156-induced ISR to B. cinerea B1301 in Arabidopsis, we assessed disease development in Col-0 and the two mutant lines at $2 \mathrm{dpi}$. Two-way ANOVA with Tukey's test was conducted to compare the differences in disease severity between AR156-treated and non-treated plants with different genotypes infected with the pathogen. As shown in Fig. 6a, B. cinerea B1301 infection caused severe disease symptoms on leaves of control plants from both Col-0 and the two mutant lines, while the symptoms 
were more severe in the mutants than in Col-0, indicating the mutant lines were more prone to the attack by B. cinerea B1301 than Col-0 (Fig. 6a). However, AR156 pretreatment caused a noticeable attenuation of disease symptoms on plants of Col-0 and the two mutants, demonstrating that AR156 induced effective ISR in both Col-0 and the mutants. Consistently, AR156-treated plants of Col-0 and target mutant lines developed significantly ( $\left.{ }^{*} P<0.01\right)$ smaller necrotic lesions on leaves than their respective control plants; on the other hand, the lesions in the target mutants were larger than those in Col-0 plants (Fig. 6b). The fungal growth rates in plants of Col-0 and the two target mutant lines were also determined by qRTPCR. In consistence with the inhibitory effect of AR156 pretreatment on the disease symptoms observed on leaf surface, the growth of $B$. cinerea B1301 at 2 dpi was significantly slower in AR156/B. cinerea than in Control/ $B$. cinerea regardless of plant lines; on the other hand, the fungal growth rate increased in target mutants than in Col-0 plants regardless of pretreatment (AR156 or Control) (Fig. 6c). Importantly, the two-way ANOVA showed a significant interactive effect of treatment and genotype, as well as a significant treatment effect demonstrated by that the overall levels of resistance to $B$. cinerea B1301 in AR156-pretreated plants significantly exceeded those in control plants across different genotypes. Taken together, the target mutant plants showed increased susceptibility to the pathogen, yet AR156 still retained the capacity to trigger ISR in them.

\section{Discussion}

Biological control is an effective strategy for protecting plants from pathogen infection. Our earlier researches demonstrated that the PGPR strain B. cereus AR156 can elicit ISR against Pst DC3000 in Arabidopsis through activating both of the SA-dependent and the JA/ET-dependent signaling pathway $[10,45]$. More recently, we found that AR156 also triggers ISR effective in controlling $B$. cinerea B1301, a necrotrophic pathogen, which is dependent on the JA/ET pathway, but not the SA pathway [28]. Moreover, documented studies have elucidated the molecular processes and the components of signaling pathways contributing to the interactions between host plants and ISR elicitors [12, 18]. We also reported that miR825 and miR825* downregulate AR156-meditated ISR against Pst [42]. On the other side, it is known that miRNAs also function to suppress plant innate immunity, as evidenced by that without pathogen infection, they inhibit plant defense responses; however, under the pathogenic stress, healthy plants express a variety of immune responses by relieving miRNAs-regulated repression [32, 42, 46]. The present study showed that AR156 pretreatment triggers ISR to B. cinerea B1301 by inhibiting miR825 and miR825 ${ }^{*}$ expression, thereby inducing the expression of their targeted defense genes in Arabidopsis.

It is known that defense-related genes are induced when plants and pathogens interact under primed conditions. PR1 gene expression was stronger in AR156/Pst samples than in Control/Pst [10, 20]. The induced defense-related gene expression has also been observed in ISR to some necrotrophic and hemibiotrophic pathogens; for example, the expression of defense genes was induced in rice plants primed by Harpophora oryzae and infected by Magnaporthe oryzae [47], in tomato plants primed by Bacillus subtilis CBR05 and infected by Erwinia carotovora subsp. Carotovora [48], and in Arabidopsis plants pretreated by $B$. cereus AR156 and infected by $B$. cinerea B1301 [28]. In line with this, it has been 
documented that enhanced $P R$ gene expression promotes defense responses to necrotrophic and hemibiotrophic pathogens $[47,48]$. In this study, the transcriptional levels of $P R 1, P R 2, P R 5$, and $P D F 1.2$ in plants with different genotypes were analysed by two-way ANOVA. On the one hand, AR156 treatment significantly stimulated their transcription and enhanced hydrogen peroxide accumulation and callose deposition in Col-0, STTM825/825*, and miR825/825* OE plants upon infection with B. cinerea B1301; on the other hand, these events were significantly stronger in the STTM $825 / 825^{*}$ transgenic plants, but weaker in the miR825/825* OE plants than those in Col-0 plants (Fig. 3). Importantly, the interaction between treatment effect and genotype effect was statistically very significant $(P<0.01)$ (Fig. 3a). Our results indicated that miR825 and miR825* played a negative regulatory role in AR156-elicited potentiation of cellular defense responses. This finding is supported by prior studies demonstrating that potentiated accumulation of hydrogen peroxide and deposition of callose are important components of plant immune responses to the attack by B. cinerea B1301 $[49,50]$.

PTI is indispensable to plant defense against $B$. cinerea B1301, the earliest events in PTI include activation of mitogen-activated protein kinases (MAPKs) [44, 51, 52]. MAPK cascade signaling is essential for plant defense against pathogen infections. In the current research, MPK3 and MPK6 phosphorylation was detected at $10 \mathrm{mpi}$ in both Control/B. cinerea and AR156/B. cinerea from plants of Col-0, miR825/825* OE line (\#50), and miR825/825* knockdown line (\#1); and during 10-60 mpi, the phosphorylation was considerably stronger in AR156/B. cinerea than in Control/B. cinerea regardless of plant line (Fig. 4a). Therefore, the treatment with AR156 enhanced MPK3 and MPK6 phosphorylation. Many studies have showed that MPK3 and MPK6 are positive mediators of plant disease resistance [5254], being critical for plant immunity to $B$. cinerea B1301 [55-57]. In the present study, the expression of FRK1 and WRKY53, two MAMP-specific early-defense marker genes, was down- and up-regulated in miR825/825* OE and knockdown lines, respectively, compared with that in Col-0 plants, which demonstrated the involvement of miR825 and miR825* in innate immunity and also consolidated our conclusion that they are negative regulators of AR156-induced ISR to B. cinerea B1301. Nonetheless, the exact function of miR825 and miR825 in sub-branches of PTI remains to be deciphered. Given that miR825 and miR825* also negatively regulate defense responses in AR156-induced ISR to Pst DC3000 [42], they might regulate plant resistance to the bacterium and the fungus by using similar mechanisms.

An $R$ gene family of the TIR-NBS-LRR class is targeted by miR825*, whose target site lies in the region that encodes the TIR domain, which is commonly shared by the proteins of TIR-NBS-LRR family [42]. miR825* may target a number of $R$ genes [42]. On the other side, miR825 targets AT5G44940, a member of F-box/RNI-like superfamily. Moreover, proteins in F-box/RNI-like superfamily are involved in protein ubiquitination; transport inhibitor response 1 (TIR1), a F-box auxin receptor, modulates auxin signaling by triggering auxin/indole-3-acetic acid (Aux/IAA) proteasomal degradation [58]. TIR1 is targeted by miR393, whose expression is activated by flg22, thus contributing to PTI [34]. Our study demonstrated that miR825 and miR825 ${ }^{\star}$ act as negative regulators of AR156-triggered ISR to B. cinerea B1301 by repressing the transcription of the targeted defense genes activated by AR156 in plants upon its attack. Therefore, we infer that miR825 might participate in AR156-induced ISR by specifically activating auxin signaling 
through protein ubiquitination. Additionally, the level of AR156-mediated ISR to B. cinerea B1301 was lower in each of the target mutants than that in wild type plants (Fig. 6). The two-way ANOVA demonstrated that the interaction between treatment effect and genotype effect was statistically significant and that the levels of resistance to B. cinerea B1301 in AR156-treated plants overall significantly surpassed those in control plants across different genotypes. Hence, these target genes may participate in AR156-induced ISR.

The present study showed that miR825 and miR825* suppress AR156-elicited ISR to B. cinerea B1301 stress, as demonstrated by the down- and up-regulation of several defense-related genes in $825 / 825^{\star}$ OE and STTM825/825* plants, respectively, compared with Col-0 plants. Moreover, STTM825/825* plants without AR156 treatment showed stronger resistance than Col-0 to a wide variety of pathogens including virus and oomycetes, which indicated that miR825 and miR825 ${ }^{*}$ also function to negatively regulate innate immunity. Therefore, the findings of this study have significant implications for effectively applying the two miRNAs to plant protection.

\section{Conclusions}

In summary, northern blotting analysis indicated that upon B. cinerea B1301 inoculation, miR825 and miR825 ${ }^{*}$ were more strongly downregulated in AR156-pretreated plants than in non-treated control plants. Furthermore, compared with Col-0, the short tandem target mimic (STTM) miR825 and miR825 (STTM825/825*) transgenic lines were more resistant to B. cinerea B1301, in contrast, miR825- and miR825 ${ }^{\star}$-overexpressing (OE) plants were more prone to it. Moreover, upon B. cinerea B1301 infection, the transcription of defense-related genes and cellular defense responses (hydrogen peroxide production and callose deposition) were faster and stronger in AR156-pretreated plants from miR825 and miR825* knockdown lines, but weaker in miR825 and miR825* OE plants than in Col-0 plants. AR156 pretreatment caused stronger phosphorylation of MPK3 and MPK6 and expression of FRK1 and WRKY53 genes upon B. cinerea B1301 inoculation in miR825 and miR825* knockdown plants than in Col-0 plants. In addition, we identified a number of $R$ genes of the TIR-NBS-LRR class as miR825* targets, which were expressed in a similar manner during AR156-triggered ISR. Furthermore, compared with Col-0, the target mutant lines showed higher susceptibility to $B$. cinerea B1301 while still expressing AR156-triggered ISR. miR825 and miR825* act as negative regulators of AR156-mediated systemic resistance to $B$. cinerea B1301 in Arabidopsis.

\section{Abbreviations}

PRRs: Pattern-recognition receptors; PAMPs: pathogen-associated molecular patterns; PTI: PAMPtriggered immunity; MAPK: mitogen-activated protein kinase; ETI: effector-triggered immunity; HR: hypersensitive response; SAR: Systemic acquired resistance; ISR: induced systemic resistance; PGPR: plant-growth-promoting rhizobacteria; SA: salicylic acid; JA: jasmonic acid; ET: ethylene; PR:

pathogenesis-related; PDF1.2: plant defensin 1.2; B. cinerea: Botrytis cinerea; sRNAs: Small RNAs; siRNAs: 
small interfering RNAs; miRNAs: microRNAs; AGO: argonaute; TuMV: Turnip mosaic virus; TMV: tobacco mosaic virus; P. capsici: Phytophthora capsici; CMV: cucumber mosaic virus; RPS5: resistance Pseudomonas syringae 5; CC: coiled-coil; Pst. Pseudomonas syringae pv. Tomato; OE: overexpressing; STTM: short tandem target mimic; dpt: days post-treatment; dpi: days post-inoculation; hpi: hours postinoculation; mpi: min post-inoculation; qRT-PCR: quantitative real time PCR; FRK1: flg22-induced receptorlike kinase 1; MAPKs: mitogen-activated protein kinases; TIR1: transport inhibitor response 1; Aux/IAA: auxin/indole-3-acetic acid

\section{Declarations}

\section{Acknowledgements}

Not applicable.

\section{Funding}

This study was financially supported by the Natural Science Foundation of Jiangsu Province of China (BK20171382) \the Fundamental Research Funds for the Central Universities (Y0201700152)هQing Lan Project of Jiangsu Province to D. D. Niu; The Research Fund for the Doctoral Program of Zaozhuang University, China (2018BS047) and the Natural Science Foundation of Shandong Province, China (ZR2019PC049) to P. P. Nie. The funders had no role in the experimental design, data analysis, decision to publish or preparation of the manuscript.

\section{Availability of data and materials}

All data supporting the conclusions of this article are included within the article (and its additional files).

\section{Authors' contributions}

DDN conceived the idea and designed the project; PPN, CC and CHJ performed the experiments; DDN, PPN, QY, JHG and HWZ analyzed and interpreted the data; DDN and PPN wrote the manuscript; all authors read and approved the final manuscript for publication.

\section{Authors' information}

All authors works/study in College of Plant Protection, Nanjing Agricultural University, Nanjing 210095, China, except Pingping Nie works College of Life Sciences, Zaozhuang University, Zaozhuang 277160, 
Shandong, China, and Qian Yin works in Institute of Botany, Jiangsu Province and Chinese Academy of Sciences, Nanjing, Jiangsu Province, 210014, China.

\section{Ethics approval and consent to participate}

Not applicable.

\section{Consent for publication}

Not applicable.

\section{Competing interests}

The authors declare that they have no competing interests.

\section{Author details}

${ }^{1}$ College of Plant Protection, Nanjing Agricultural University, Nanjing 210095, China. ${ }^{2}$ Key Laboratory of Integrated Management of Crop Diseases and Pests (Ministry of Education), Nanjing 210095, China.

${ }^{3}$ Institute of Botany, Jiangsu Province and Chinese Academy of Sciences, Nanjing, Jiangsu Province, 210014, China. ${ }^{4}$ College of Life Sciences, Zaozhuang University, Zaozhuang 277160, Shandong, China.

\section{References}

1. Chisholm ST, Coaker G, Day B, Staskawicz BJ. Host-microbe interactions: Shaping the evolution of the plant immune response. Cell. 2006;124:803-814.

2. Jones JD,Dangl JL. The plant immune system. Nature. 2006;444:323-329.

3. Ichimura K, Shinozaki K, Tena G, Sheen J, Henry Y, Champion A, Kreis M, Zhang SQ, Hirt H, Wilson C, Heberle BE, Ellis BE, Morris PC, Innes RW, Ecker JR, Scheel D, Klessig DF, Machida Y, Mundy J, Ohashi Y, Walker JC, Grp M. Mitogen-activated protein kinase cascades in plants: a new nomenclature. Trends in Plant Science. 2002;7:301-308.

4. Altenbach D, Robatzek S. Pattern recognition receptors: From the cell surface to intracellular dynamics. Molecular Plant-Microbe Interactions. 2007;20:1031-1039.

5. Schwessinger B, Zipfel C. News from the frontline: recent insights into PAMP-triggered immunity in plants. Current Opinion in Plant Biology. 2008;11:389-395. 
6. Padmanabhan C, Zhang XM, Jin HL. Host small RNAs are big contributors to plant innate immunity. Current Opinion in Plant Biology. 2009;12:465-472.

7. Weiberg A, Wan M, Lin FM, Zhao HW, Zhang ZH, Kaloshian I, Huang HD, Jin HL. Fungal small RNAs suppress plant immunity by Hijacking host RNA interference pathways. Science. 2013;342:118-123.

8. Katiyar AS, Jin HL. Role of small RNAs in host-microbe interactions. Annual Review of Phytopathology. 2010;48:225-246.

9. Goodman TP, Behn R, Camenen Y, Coda S, Fable E, Henderson MA, Nikkola P, Rossel J, Sauter O, Scarabosio A, Zucca C, Alberti S, Amorim P, Andrebe Y, Appert K, Arnoux G, Bortolon A, Bottino A, Chavan R, Condrea I, Droz E, Duval BP, Etienne P, Fasel D, Fasoli A, Gulejova B, Hogge JP, Horacek J, Isoz PF, Joye B, Karpushov A, Kim SH, Klimanov I, Lavanchy P, Lister JB, Llobet X, Madeira T, Magnin JC, Marinoni A, Marletaz B, Marmillod P, Martin Y, Martynov A, Maslov M, Mayor JM, Moret JM, Muck A, Paris PJ, Pavlov I, Perez A, Pitts RA, Pochelon A, Porte L, Schlatter C, Schombourg K, Shidara H, Siegrist M, Siravo U, Sushkov AV, Tonetti G, Tran MQ, Weisen H, Wischmeier M, Zabolotsky A, Zhuang G, Zhuchkova A. Safety factor profile requirements for electron ITB formation in TCV. Plasma Physics and Controlled Fusion. 2005;47:B107-B120.

10. Niu DD, Liu HX, Jiang CH, Wang YP, Wang QY, Jin HL, Guo JH. The plant growth-promoting rhizobacterium Bacillus cereus AR156 induces systemic resistance in Arabidopsis thaliana by simultaneously activating salicylate- and jasmonate/ethylene-dependent signaling pathways. Molecular Plant-Microbe Interactions. 2011;24:533-542.

11. Walters DR, Ratsep J, Havis ND. Controlling crop diseases using induced resistance: challenges for the future. Journal of Experimental Botany. 2013;64:1263-1280.

12. Pieterse $C M$, Zamioudis $C$, Berendsen RL, Weller DM, Van Wees SC, Bakke PA. Induced systemic resistance by beneficial microbes. Annual Review of Phytopathology. 2014;52:347-375.

13. Mishra MK, Chaturvedi P, Singh R, Singh G, Sharma LK, Pandey V, Kumari N, Misra P. Overexpression of WSSGTL 1 gene of Withania somnifera enhances salt tolerance, heat tolerance and cold acclimation ability in transgenic Arabidopsis plants. Plos One. 2013;8:e63064.

14. Conrath U, Pieterse CM, Mauch MB. Priming in plant-pathogen interactions. Trends in Plant Science. 2002;7:210-216.

15. Durrant WE, Dong X. Systemic acquired resistance. Annual Review of Phytopathology. 2004;42:185209.

16. Choudhary DK, Johri BN. Interactions of Bacillus spp. and plants with special reference to induced systemic resistance (ISR). Microbiological Research. 2009;164:493-513.

17. Van der Ent S, Verhagen BW, Van Doorn R, Bakker D, Verlaan MG, Pel MJ, Joosten RG, Proveniers MC, Van Loon LC, Ton J, Pieterse CM. MYB72 is required in early signaling steps of rhizobacteria-induced systemic resistance in Arabidopsis. Plant Physiology. 2008;146:1293-1304.

18. Van Loon LC, Bakker PA, Pieters CM. Systemic resistance induced by rhizosphere bacteria. Annual Review of Phytopathology. 1998;36:453-483. 
19. Heil M, Bostock RM. Induced systemic resistance (ISR) against pathogens in the context of induced plant defences. Ann Bot. 2002;89:503-512.

20. Ahn IP, Lee SW, Suh SC. Rhizobacteria-induced priming in Arabidopsis is dependent on ethylene, jasmonic acid, and NPR1. Molecular Plant-Microbe Interactions. 2007;20:759-768.

21. Rahman A, Uddin W, Wenner NG. Induced systemic resistance responses in perennial ryegrass against Magnaporthe oryzae elicited by semi-purified surfactin lipopeptides and live cells of Bacillus amyloliquefaciens. Mol Plant Pathol. 2014;16:546-558.

22. Yedidia I, Shoresh M, Kerem Z, Benhamou N, Kapulnik Y, Chet I. Concomitant induction of systemic resistance to Pseudomonas syringae pv. lachrymans in cucumber by Trichoderma asperellum (T203) and accumulation of phytoalexins. Appl Environ Microbiol. 2003;69:7343-7353.

23. Glazebrook J. Genes controlling expression of defense responses in Arabidopsis - 2001 status. Current Opinion in Plant Biology. 2001;4:301-308.

24. Ward ER, Uknes SJ, Williams SC, Dincher SS, Wiederhold DL, Alexande DC, Ahlgoy P, Metraux JP, Ryals JA. Coordinate gene activity in response to agents that Induce systemic acquired-resistance. Plant Cell. 1991;3:1085-1094.

25. Van Loon LC. Induced resistance in plants and the role of pathogenesis-related proteins. European Journal of Plant Pathology. 1997;103:753-765.

26. Van Oosten VR, Bodenhausen N, Reymond P, Van Pelt JA, Van Loon LC, Dicke M, Pieterse CM. Differential effectiveness of microbially induced resistance against herbivorous insects in Arabidopsis. Molecular Plant-Microbe Interactions. 2008;21:919-930.

27. Pieterse CM, Van Wees SC, Ton J, Van Pelt JA, Van Loon LC. Signalling in rhizobacteria-induced systemic resistance in Arabidopsis thaliana. Plant Biology. 2002;4:535-544.

28. Nie PP, Li X, Wang SE, Guo JH, Zhao HW, Niu DD. Induced systemic resistance against Botrytis cinerea by Bacillus cereus AR156 through a JA/ET- and NPR1-dependent signaling pathway and activates PAMP-triggered immunity in Arabidopsis. Frontiers in Plant Science. 2017;8:238.

29. Salas-Marina MA, Silva-Flores MA, Uresti-Rivera EE, Castro-Longoria E, Herrera-Estrella A, CasasFlores S. Colonization of Arabidopsis roots by Trichoderma atroviride promotes growth and enhances systemic disease resistance through jasmonic acid/ethylene and salicylic acid pathways. European Journal of Plant Pathology. 2011;131:15-26.

30. Jin HL. Endogenous small RNAs and antibacterial immunity in plants. Febs Letters. 2008;582:26792684.

31. Pumplin N, Voinnet O. RNA silencing suppression by plant pathogens: defence, counter-defence and counter-counter-defence. Nature Reviews Microbiology. 2013;11:745-760.

32. Seo JK, Wu JG, Lii YF, Li Y, Jin HL. Contribution of small RNA pathway components in pant immunity. Molecular Plant-Microbe Interactions. 2013;26:617-625.

33. Niu DD, Lii YE, Chellappan P, Lei L, Peralta K, Jiang CH, Guo JH, Coaker G, Jin HL. miRNA863-3p sequentially targets negative immune regulator ARLPKs and positive regulator SERRATE upon bacterial infection. Nature Communications. 2016a;7:11324. 
34. Navarro L, Dunoyer P, Jay F, Arnold B, Dharmasiri N, Estelle M, Voinnet O, Jones JD. A plant miRNA contributes to antibacterial resistance by repressing auxin signaling. Science. 2006;312:436-439.

35. Li Y, Zhang QQ, Zhang JG, Wu L, Qi YJ, Zhou JM. Identification of microRNAs involved in pathogenassociated molecular pattern-triggered plant innate immunity. Plant Physiology. 2010;152:22222231.

36. Zhang X, Zhao H, Gao S, Wang WC, Katiyar Agarwal S, Huang HD, Raikhel N, Jin H. Arabidopsis Argonaute 2 regulates innate immunity via miRNA393(*)-mediated silencing of a Golgi-localized SNARE gene, MEMB12. Mol Cell. 2011;42:356-366.

37. Zhai JX, Jeong DH, De Paoli E, Park S, Rose BD, Li YP, Gonzalez AJ, Yan Z, Kitto SL, Grusak MA, Jackson SA, Stacey G, Cook DR, Green PJ, Sherrier DJ, Meyers BC. MicroRNAs as master regulators of the plant NB-LRR defense gene family via the production of phased, trans-acting siRNAs. Genes and Development. 2011;25:2540-2553.

38. Li F, Pignatta D, Bendix C, Brunkard JO, Cohn MM, Tung J, Sun HY, Kumar P, Baker B. MicroRNA regulation of plant innate immune receptors. Proceedings of the National Academy of Sciences of the United States of America. 2012;109:1790-1795.

39. He XF, Fang YY, Feng L, Guo HS. Characterization of conserved and novel microRNAs and their targets, including a TuMV-induced TIR-NBS-LRR class R gene-derived novel miRNA in Brassica. Febs Letters. 2008;582:2445-2452.

40. Shivaprasad PV, Chen HM, Patel K., Bond DM, Santos BA, Baulcombe DC. A microRNA superfamily regulates nucleotide binding site-leucine-rich repeats and other mRNAs. Plant Cell. 2012;24:859-874.

41. Boccara M, Sarazin A, Thiebeauld O, Jay F, Voinnet O, Navarro L, Colot V. The Arabidopsis miR472RDR6 silencing pathway modulates PAMP- and effector-triggered immunity through the posttranscriptional control of disease resistance genes. Plos Pathogens. 2014;10:e1003883.

42. Niu DD, Xia J, Jiang CH, Qi BB, Ling XY, Lin SY, Zhang WX, Guo JH, Jin HL, Zhao HW. Bacillus cereus AR156 primes induced systemic resistance by suppressing miR825/825*and activating defenserelated genes in Arabidopsis. Journal of Integrative Plant Biology. 2016b;58:426-439.

43. Asai T, Tena G, Plotnikova J, Willmann MR, Chiu WL, Gomez L, Boller T, Ausubel FM, Sheen J. MAP kinase signalling cascade in Arabidopsis innate immunity. Nature. 2002;415:977-983.

44. Singh P, Kuo YC, Mishra S, Tsa CH, Chien CC, Chen CW, Desclos-Theveniaun M, Chu PW, Schulze B, Chinchilla D, Boller T, Zimmerli L. The lectin receptor kinase-VI. 2 is required for priming and positively regulates Arabidopsis pattern-triggered immunity. Plant Cell. 2012;24:1256-1270.

45. Niu DD, Wang CJ, Guo YH, Jiang CH, Zhang WZ, Wang YP, Guo JH. The plant growth-promoting rhizobacterium Bacillus cereus AR156 induces resistance in tomato with induction and priming of defence response. Biocontrol Science and Technology. 2012;22:991-1004.

46. Ruiz-Ferrer V, Voinnet O. Roles of plant small RNAs in biotic stress responses. Annual Review of Plant Biology. 2009;60:485-510.

47. Su ZZ, Mao LJ, Li N, Feng XX, Yuan ZL, Wang LW, Lin FC, Zhang CL. Evidence for biotrophic lifestyle and biocontrol potential of dark septate endophyte Harpophora oryzae to Rice Blast Disease. Plos 
One. 2013;8.

48. Chandrasekaran M, Chun SC. Expression of PR-protein genes and induction of defense-related enzymes by Bacillus subtilis CBR05 in tomato (Solanum lycopersicum) plants challenged with Erwinia carotovora subsp carotovora. Bioscience Biotechnology and Biochemistry. 2016;80:22772283.

49. Mengiste T. Plant immunity to necrotrophs. Annual Review of Phytopathology. 2012;50:267-294.

50. Schwessinger B, Ronald PC. Plant innate immunity: perception of conserved microbial signatures. Annual Review of Plant Biology. 2012;63:451-482.

51. Eckardt NA. BIK1 function in plant growth and defense signaling. Plant Cell. 2011;23:2806-2806.

52. Meng XZ, Zhang SQ. MAPK cascades in plant disease resistance signaling. Annual Review of Phytopathology. 2013;51:245-266.

53. Schweighofer A, Meskiene I. Regulation of stress hormones jasmonates and ethylene by MAPK pathways in plants. Molecular Biosystems. 2008;4:799-803.

54. Tena G, Boudsocq M, Sheen J. Protein kinase signaling networks in plant innate immunity. Current Opinion in Plant Biology. 2011;14:519-529.

55. Ren DT, Liu YD, Yang KY, Han L, Mao GH, Glazebrook J, Zhang SQ. A fungal-responsive MAPK cascade regulates phytoalexin biosynthesis in Arabidopsis. Proceedings of the National Academy of Sciences of the United States of America. 2008;105:5638-5643.

56. Han L, Li GJ, Yang KY, Mao GH, Wang RQ, Liu YD, Zhang SQ. Mitogen-activated protein kinase 3 and 6 regulate Botrytis cinerea-induced ethylene production in Arabidopsis. Plant Journal. 2010;64:114127.

57. Mendez BA, Calderon VC, Ibarra LE, Raya GJ, Ramirez CE, Molina TJ, Guevara GA, Lopez BJ, Herrera EL. Alkamides activate jasmonic acid biosynthesis and signaling pathways and confer resistance to Botrytis cinerea in Arabidopsis thaliana. Plos One. 2011;6.

58. Yang XY, Wang LC, Yuan DJ, Lindsey K, Zhang XL. Small RNA and degradome sequencing reveal complex miRNA regulation during cotton somatic embryogenesis. Journal of Experimental Botany. 2013;64:1521-1536.

59. Lu S, Chen LL, Tao K, Sun NN, Wu YR, Lu XX, Wang YC, Dou DL. Intracelluar and extracelluar phosphatidylinositol 3-phosphate produced by Phytophthora species is important for infection. Molecular Plant. 2013;6:1592-1604.

60. Yan H, Zhu JH, Chen T, Zhang L. MicroRNA profiling identifies miR-146a and miR-29a and their target genes CD4OL and LPL in the regulation of oxLDL-treated dendritic cell maturation, inflammatory response and lipid uptake. European Heart Journal. 2011;32:27-28.

\section{Figures}



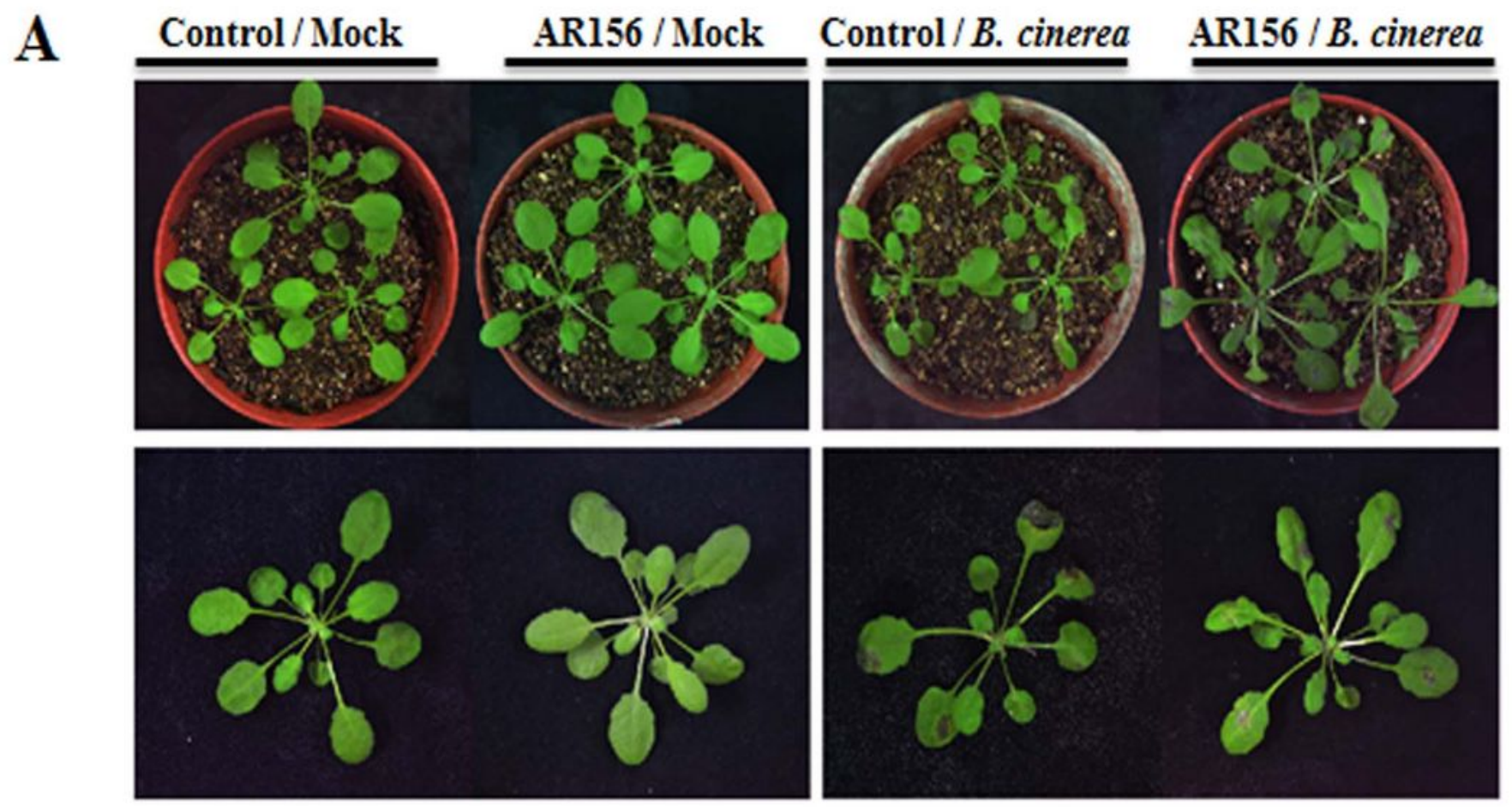

B

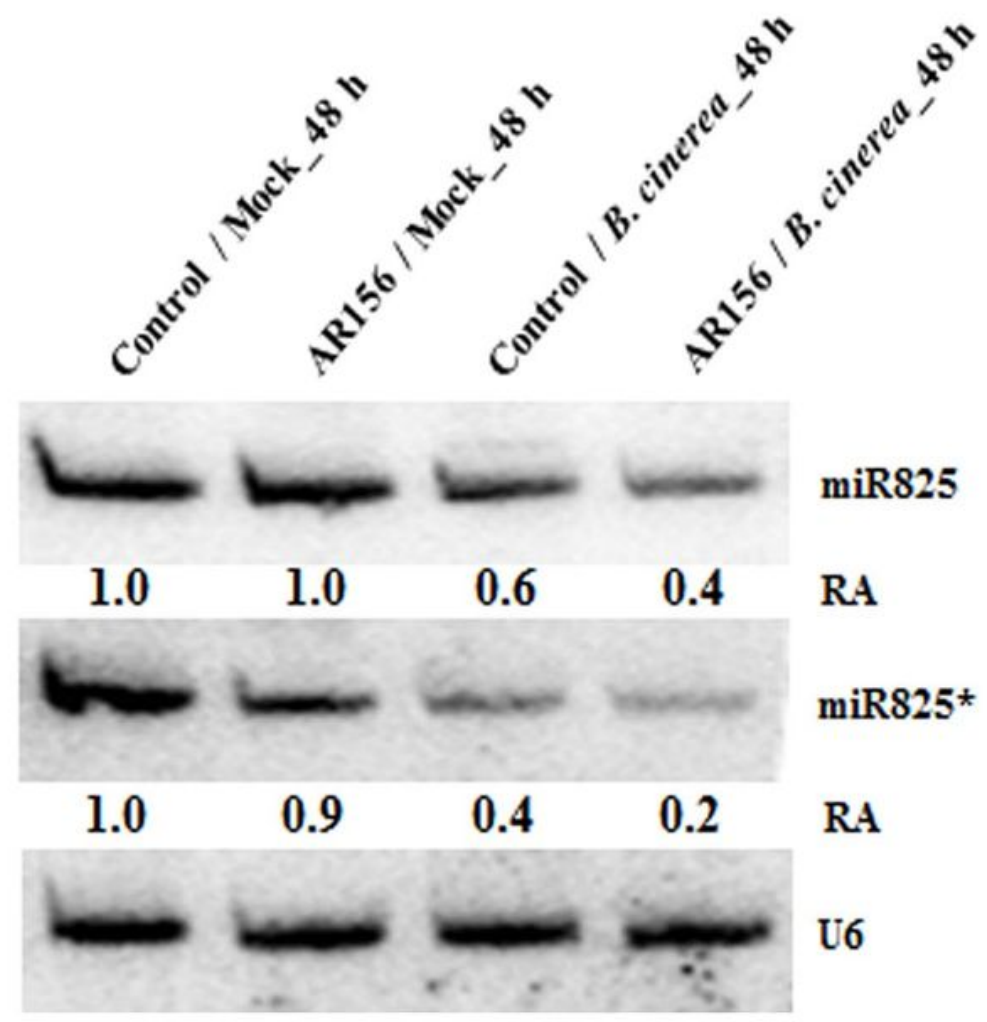

\section{Figure 1}

miR825 and miR825* expression was suppressed in AR156-elicited ISR to B. cinerea in Arabidopsis. Three-week-old Arabidopsis Col-0 plants were pretreated with AR156 at $5 \times 108 \mathrm{CFU} / \mathrm{ml}$ or $0.85 \% \mathrm{NaCl}$ (the control) as a root drench; at 7 days post treatment (dpt), challenge inoculation was carried out by dropping $10 \mu \mathrm{l}$ of a B. cinerea B1301 spore suspension at $1 \times 106$ spores $/ \mathrm{ml}$ or $\mathrm{H} 2 \mathrm{O}$ (mock) on the midvein of each side of a leaf. a At 48 hours post inoculation (hpi), disease symptoms on leaves were 
observed and photos were taken. b miR825 and miR825* expression in different treatments was detected by northern blotting. Total RNA was extracted from leaves of Arabidopsis Col-0 plants in four treatments (Control/Mock, AR156/Mock, Control/B. cinerea, and AR156/B. cinerea) at $48 \mathrm{hpi}$. RNA blots were probed with DNA oligonucleotides complementary to miR825 or miR825*. U6 served as a loading control. Relative abundance (RA) levels are indicated. The experiments were repeated three times, which yielded similar results.

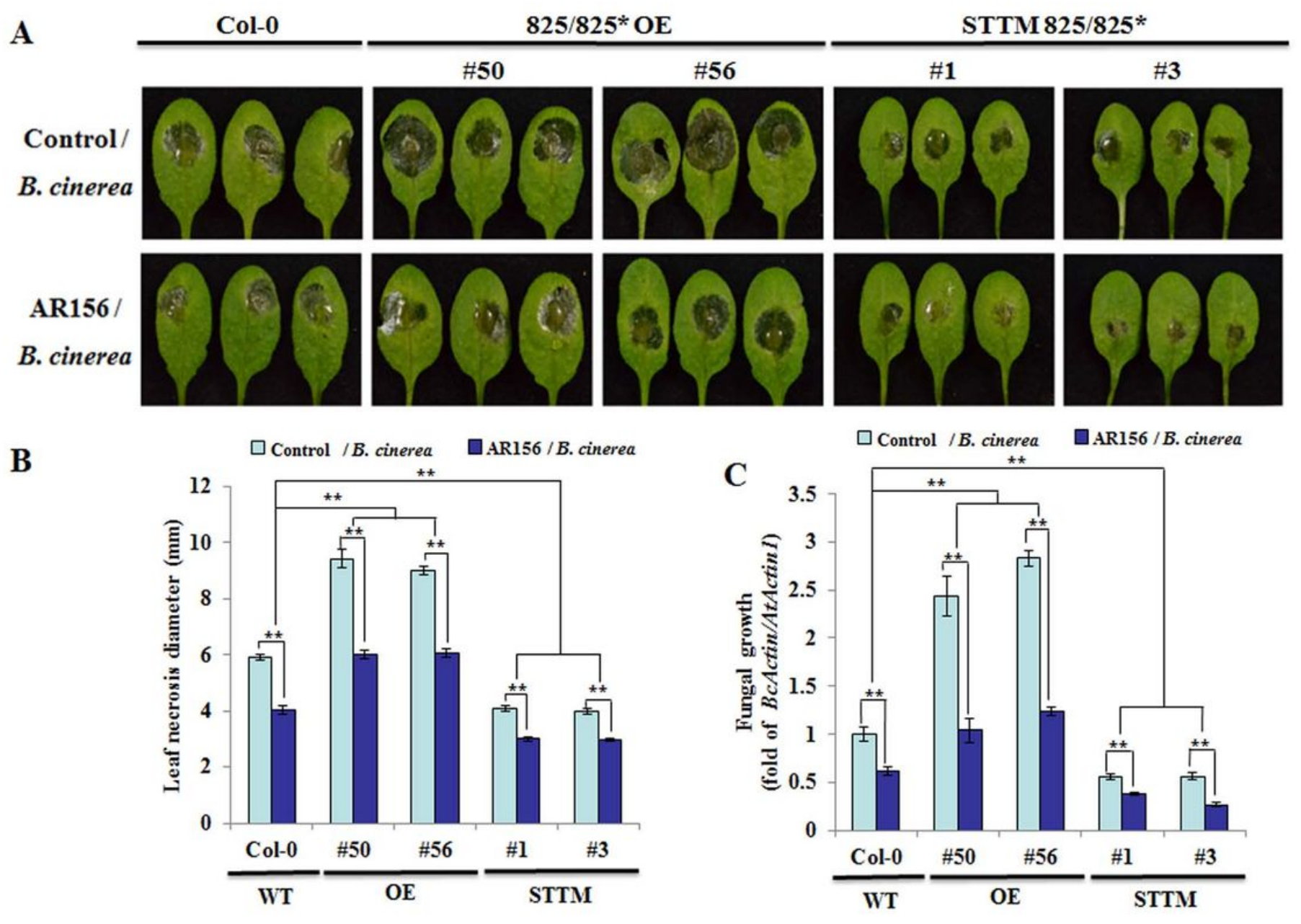

Figure 2

Plant resistance to B. cinerea increased in STTM825/825* lines, but decreased in miR825/825* OE plants in comparison with that in Col-0. Three-week-old Arabidopsis plants from Col-0, miR825/825* OE lines (\#50 and \#56), and STTM825/825* lines (\#1 and \#3) were pretreated with AR156 at $5 \times 108 \mathrm{CFU} / \mathrm{ml}$ or $0.85 \% \mathrm{NaCl}$ (Control) as a root drench; at $7 \mathrm{dpt}$, all plants were infected with B. cinerea B1301 by dropping $10 \mu$ l of its spore suspension $(1 \times 106$ spores $/ \mathrm{ml})$ on the midvein of each side of a leaf. a Disease symptoms on leaves of each tested plant line at $2 \mathrm{dpi}$. b Necrotic lesions were evaluated at $2 \mathrm{dpi}$ by determining their average diameter on one leaf per plant, with a total of three plants evaluated for each sample. $c$ In planta fungal growth in leaves of each tested plant line. The growth rate of B. cinerea B1301 was determined by simultaneously quantifying the transcripts of B. cinerea actin gene (BcActin) and the Arabidopsis actin 1 gene (AtActin1). Relative fungal growth rate was indicated by BcActin/AtActin1, the 
ratio of BcActin to AtActin1. Two-way ANOVA showed significant effects of genotype $(P<0.01)$ and treatment $(P<0.01)$ and a significant interactive effect $(P<0.01)$; $* \star, P<0.01$. The data are presented as mean \pm SD from three biological replicates. The experiments were repeated three times, bringing similar results.

A
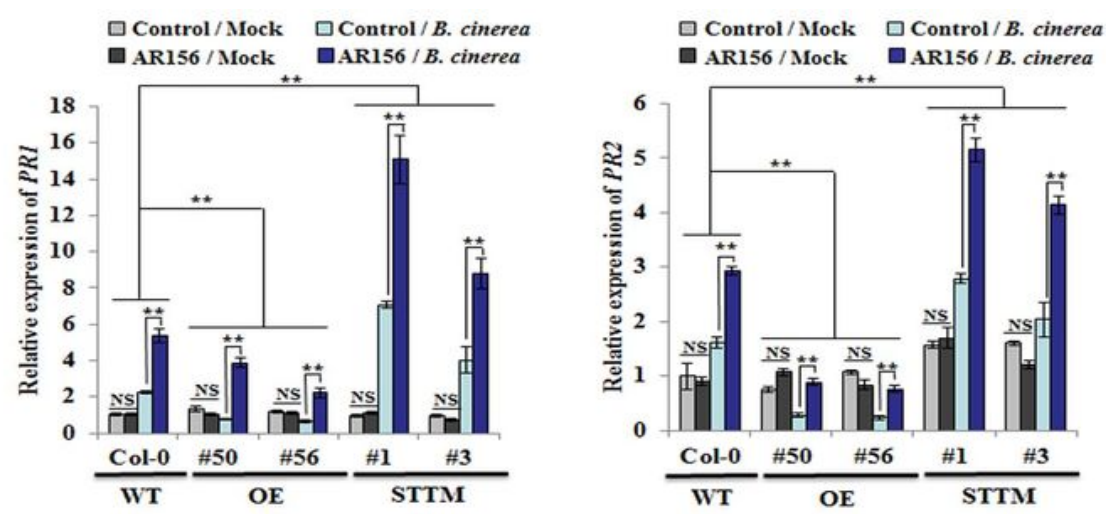

$\square$ Control/Mock $\square$ Control/B. cinerea
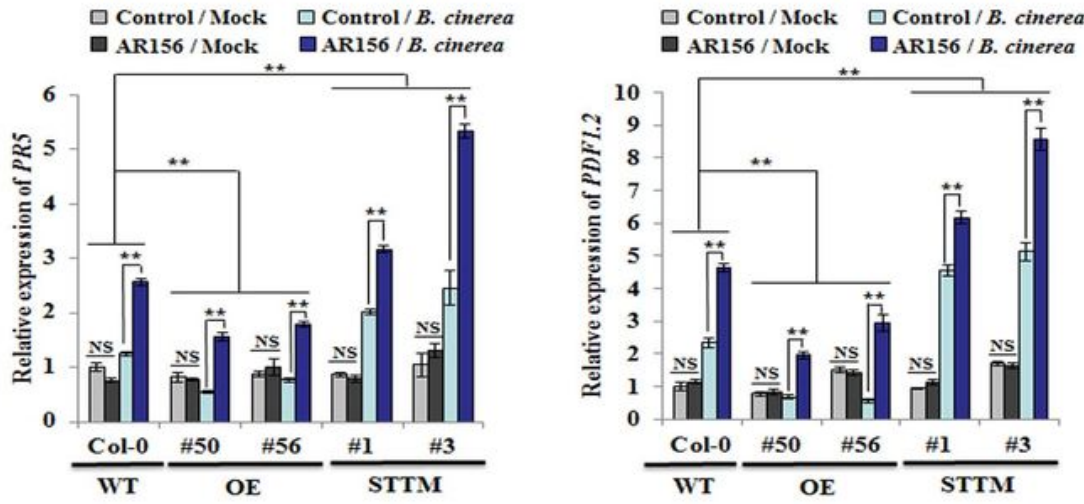

B

\begin{tabular}{|c|c|c|c|c|c|c|c|}
\hline 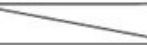 & Col-0 & OE \#50 & OE \#56 & STTM $\# 1$ & STTM \#3 & \multirow{3}{*}{12 hpi } & \multirow{5}{*}{ 兽竞 } \\
\hline $\begin{array}{l}\text { Control/ } \\
\text { B. cinerea }\end{array}$ & & & & & & & \\
\hline $\begin{array}{c}\text { AR156/ } \\
\text { B. cinerea }\end{array}$ & & & & & & & \\
\hline $\begin{array}{l}\text { Control/ } \\
\text { B. cinerea }\end{array}$ & & & & & & \multirow{2}{*}{24 hpi } & \\
\hline $\begin{array}{c}\text { AR156/ } \\
\text { B. cinerea }\end{array}$ & & & & & & & \\
\hline $\begin{array}{l}\text { Control/ } \\
\text { B. cinerea }\end{array}$ & & & & & & \multirow{2}{*}{12 hpi } & \multirow{4}{*}{ 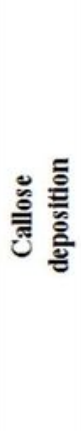 } \\
\hline $\begin{array}{c}\text { AR156/ } \\
\text { B. cinerea }\end{array}$ & a: & & is & & 8 & & \\
\hline $\begin{array}{l}\text { Control/ } \\
\text { B. cinerea }\end{array}$ & 8 & & & & & \multirow[b]{2}{*}{24 hpi } & \\
\hline $\begin{array}{c}\text { AR156/ } \\
\text { B. cinerea }\end{array}$ & & $\therefore$ & & & & & \\
\hline
\end{tabular}

Figure 3 
miR825 and miR825* affected defense gene expression and cellular defense responses in Arabidopsis plants primed by AR156. Roots of three-week-old plants from Col-0, miR825/825* OE lines (\#50 and \#56), and STTM825/825* lines (\#1 and \#3) were drenched with AR156 at $5 \times 108 \mathrm{CFU} / \mathrm{ml}$ or $0.85 \% \mathrm{NaCl}$ (Control). After 7 days, the leaves of these plants were inoculated with a B. cinerea B1301 spore suspension $(1 \times 106$ spores/ml) or $\mathrm{H} 2 \mathrm{O}$ (Mock), which were collected at 12, 24 and 48 hpi. a Transcription of defense-related genes upon the attack by B. cinerea B1301. Using qRT-PCR, transcriptional levels of defense genes in leaves collected at $48 \mathrm{hpi}$ were examined and normalized with that of AtActin1. The data are presented as mean \pm SD from three biological replicates. The assay was repeated three times, yielding similar results. Two-way ANOVA showed significant effects of genotype on the expression of PR1, PR2, PR5, or PDF1.2 ( $<0.01$ for the differences between WT and OE, and also for those between WT and STTM); a significant treatment effect $(P<0.01)$ and a significant interactive effect $(P<0.01)$ on the expression of each tested gene; NS, not significant; $* \star, P<0.01$. b Hydrogen peroxcide accumulation and callose deposition in situ detected at 12 and 24 hpi in leaves inoculated with $B$. cinerea B1301. Accumulation of $\mathrm{H} 2 \mathrm{O} 2$ was examined by DAB staining; with aniline blue staining, callose deposition was visible under light and epifluorescence microscopes attached with a UV excitation filter. 
A

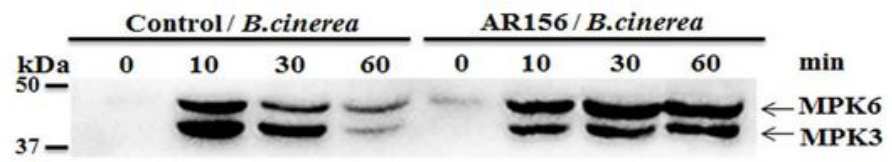

Col-0

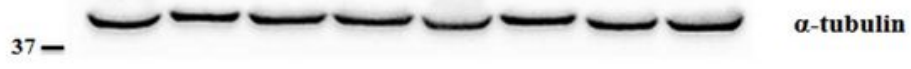

B

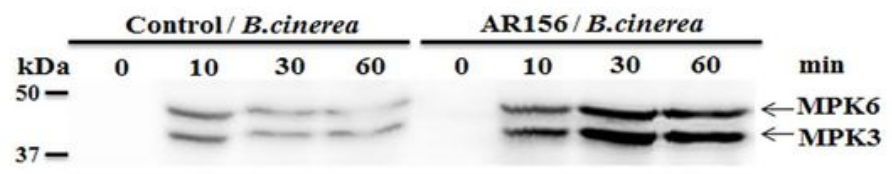

OE \#50

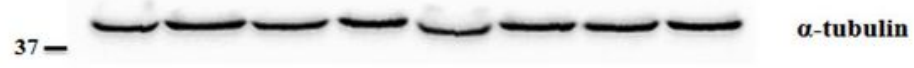

C

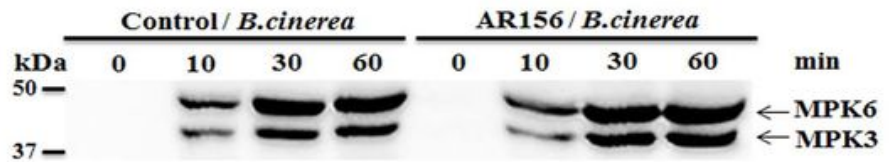

STTM \#1

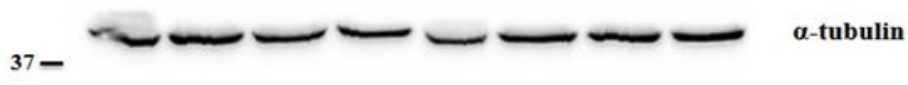

D

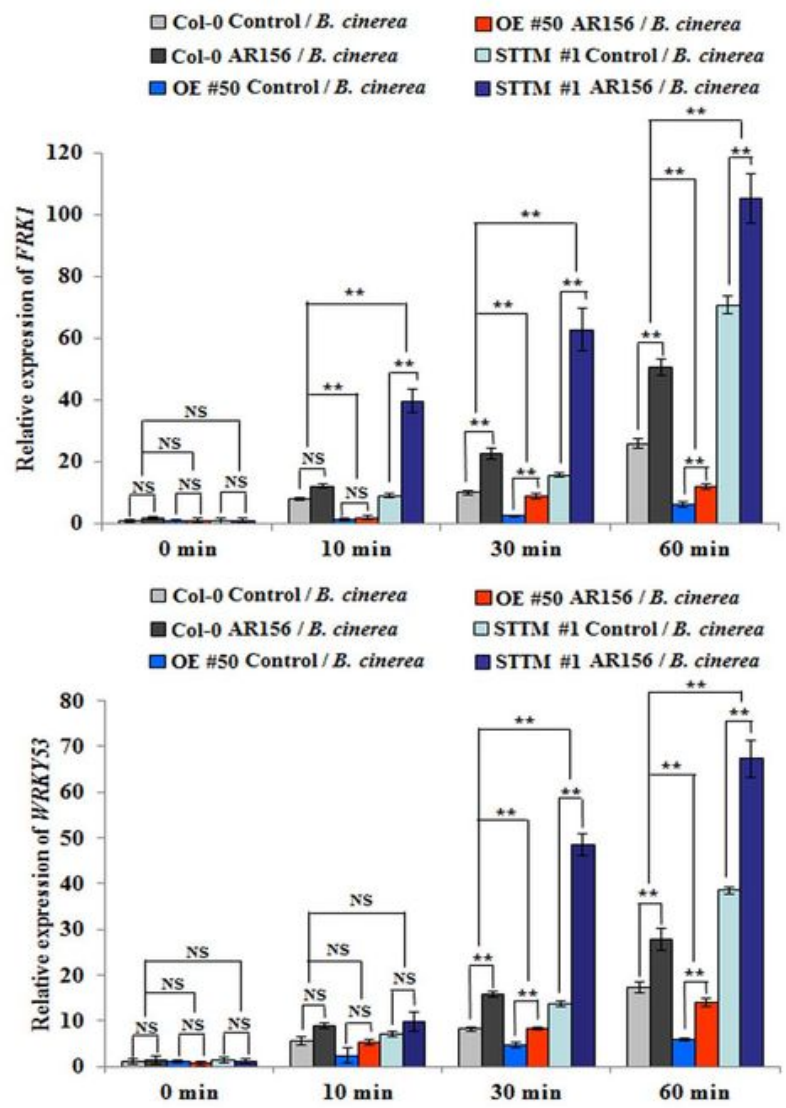

\section{Figure 4}

miR825 and miR825* affected the activation of PTI components in AR156-primed plants upon pathogen attack. Three-week-old Arabidopsis plants from Col-0, miR825/825* OE line (\#50), and STTM825/825* transgenic line (\#1) were pretreated with AR156 at $5 \times 108 \mathrm{CFU} / \mathrm{ml}$ or $0.85 \% \mathrm{NaCl}$ (the control) as a root drench. At 7 dpt, they were challenge inoculated with B. cinerea B1301 as depicted above at 0, 10, 30, and 60 mpi. a-c Leaves of each tested plant line were collected at 0, 10, 30, and 60 mpi; and phosphorylated 
MPK3 and MPK6 were detected by Western blotting, using a-tubulin as an equal loading control. $d$ The transcriptional levels of FRK1 and WRKY53 in Col-0, miR825/825* OE line (\#50), and STTM825/825* transgenic line (\#1) were evaluated by means of real-time RT-PCR. Leaves were sampled at $0,10,30$ and $60 \mathrm{mpi}$. AtActin1 mRNA was included as an internal control. Two-way ANOVA showed significant effects of genotype on the expression of FRK1 and WRKY53 ( $P<0.01$ for the differences between WT and OE, and also for those between WT and STTM); a significant treatment effect $(P<0.01)$ and a significant interactive effect $(P<0.01)$ on the expression of each tested gene; NS, not significant; $* \star, P<0.01$. The data are presented as mean \pm SD from three biological replicates. The assays were repeated three times, bringing similar results. 
$\mathbf{A}$
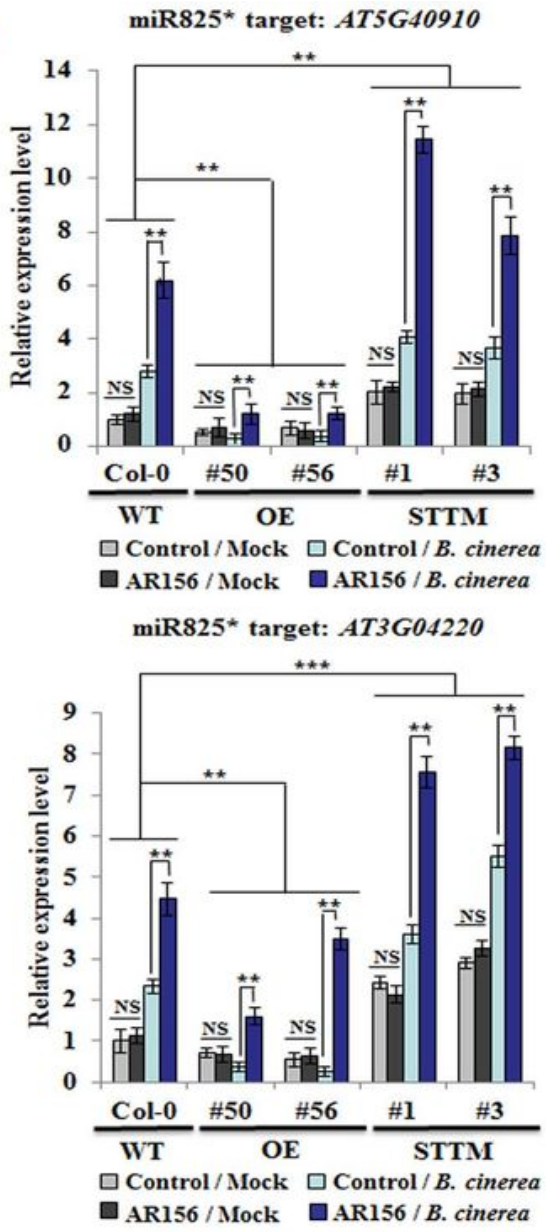

B
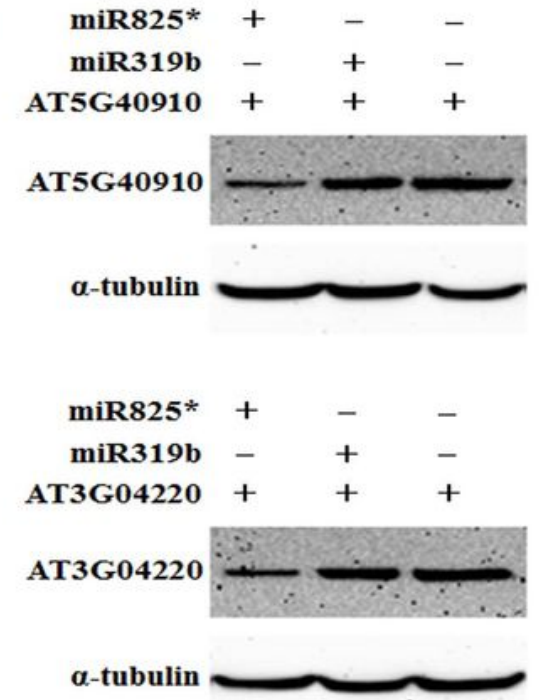

miR825* target: $A T 5 G 38850$

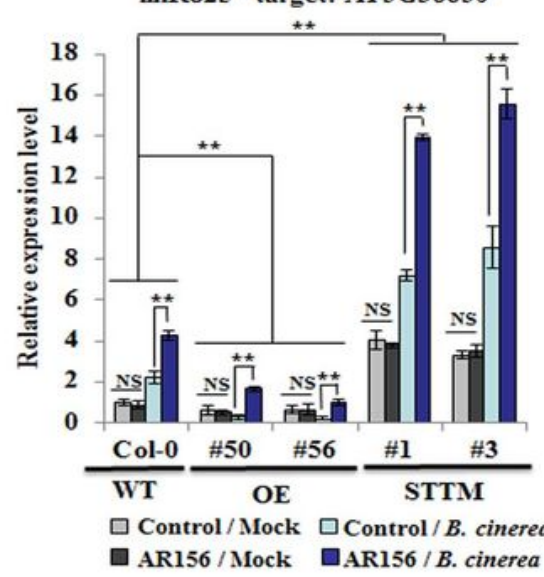

miR825 target: AT5G44940
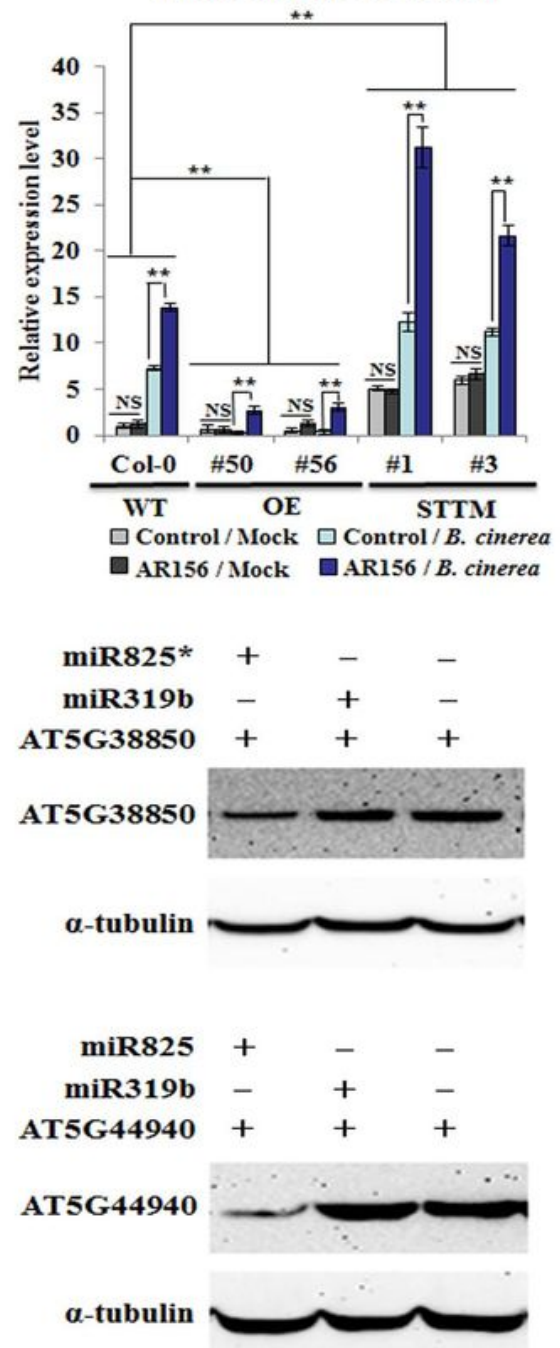

\section{Figure 5}

Target genes of miR825 and miR825* were expressed in a similar manner in AR156-induced ISR. a Expression profiles of AT5G40910, AT5G38850, and AT3G04220 (miR825* targets), and AT5G44940 (a miR825 target) in Col-0, miR825/825* OE lines (\#50 and \#56), and STTM825/825* transgenic lines (\#1 and \#3) during AR156-triggered ISR. Three-week-old plants were pretreated with AR156 at $5 \times 108 \mathrm{CFU} / \mathrm{ml}$ or $0.85 \% \mathrm{NaCl}$ (the control) as a root drench; at $7 \mathrm{dpt}$, leaves were challenge inoculated as depicted 
above. At $48 \mathrm{hpi}$, total RNA was extracted from leaves. The data are presented as mean \pm SD from three biological replicates. Two-way ANOVA indicated significant effects of genotype on the expression of AT5G40910, AT5G38850, and AT5G44940 ( $P<0.01$ for the differences between WT and OE, and also for those between WT and STTM) and on AT3G04220 expression $(P<0.01$ and $P<0.001$ for the differences between WT and OE, and between WT and STTM, respectively); a significant treatment effect $(P<0.01)$ and a significant interactive effect $(P<0.01)$ on the expression of each tested gene; NS, not significant; **, $\mathrm{P}<0.01$; ***, $\mathrm{P}<0.001$. b Co-expression of miR825/miR825* and the four target proteins (AT5G40910, AT5G38850, AT3G04220, and AT5G44940) in a transient expression system analysed by Western blotting. Through Agrobacterium-mediated transformation, the target proteins and miR825/825* or an irrelevant miRNA (miR319b) were co-expressed in Nicotiana benthmiana and detected at 2 dpi by using an anti-HA antibody. a-tubulin served as an equal loading control. The assays were repeated three times with similar results obtained.

$\mathbf{A}$

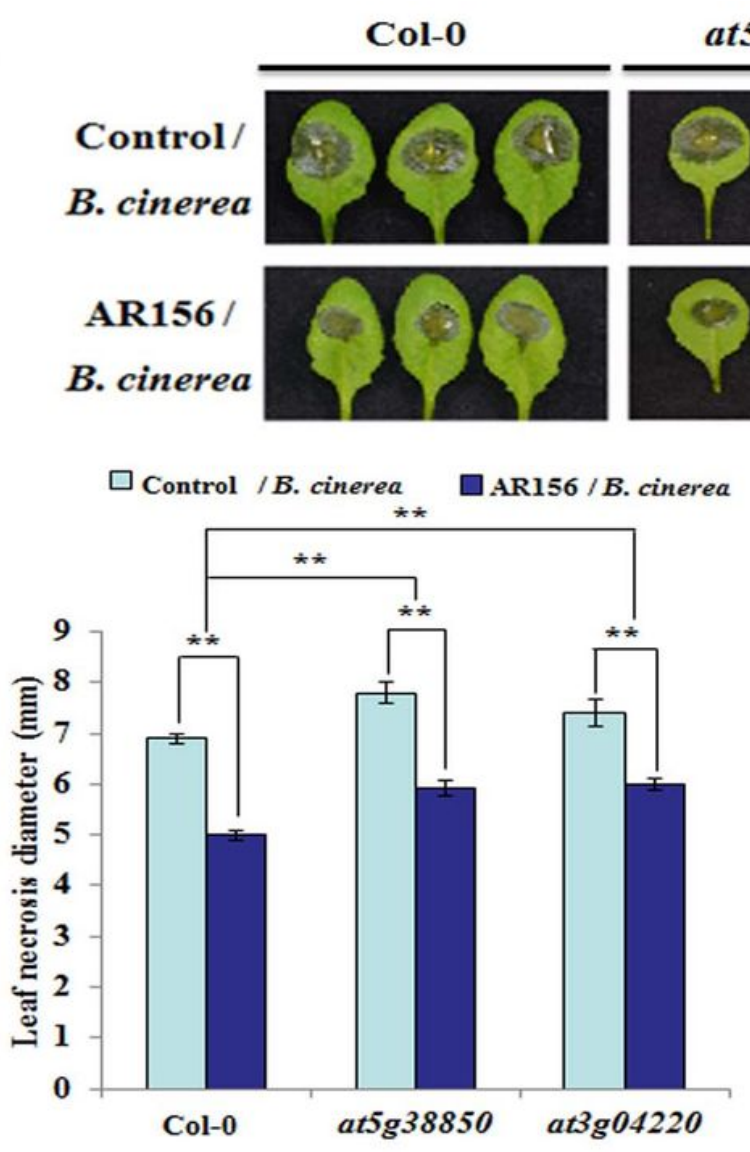

at5g38850

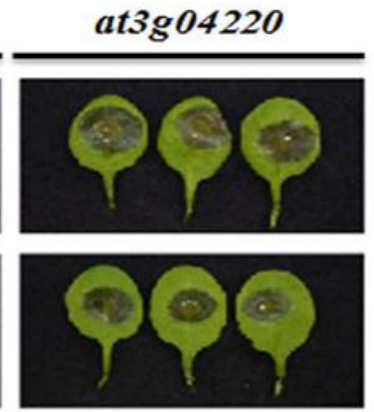

B

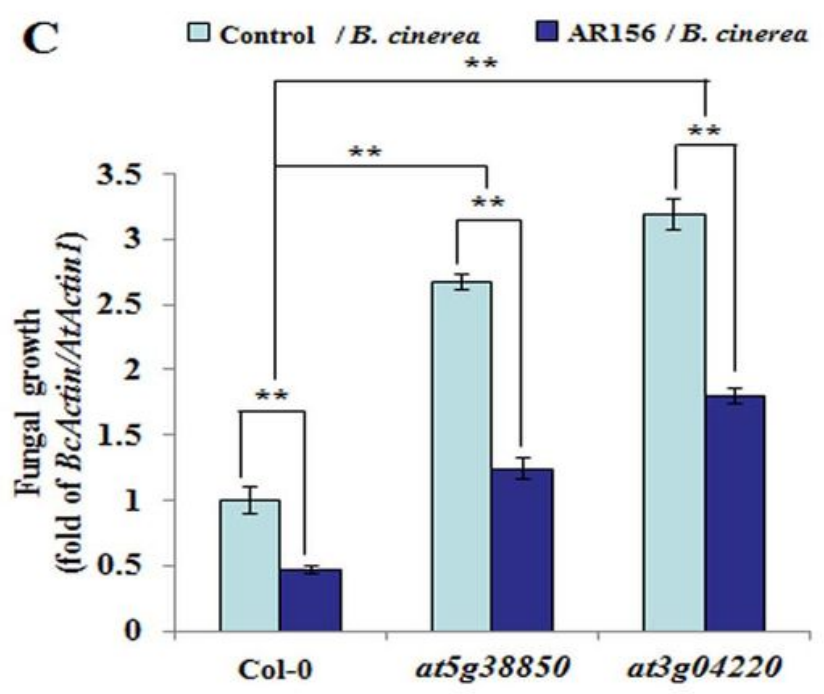

\section{Figure 6}

Plants silencing miR825/825* target genes were more prone to the attack by B. cinerea. Three-week-old plants of Arabidopsis Col-0 and miR825/825* target mutant lines (at5g38850, at3g04220) were pretreated with $\mathrm{AR} 156$ at $5 \times 108 \mathrm{CFU} / \mathrm{ml}$ or $0.85 \% \mathrm{NaCl}$ (the control) as a root drench. All plants were challenge inoculated at $7 \mathrm{dpt}$ as described above. a Disease symptoms developed at $2 \mathrm{dpi}$ on leaves of each tested plant line. b Necrotic lesions were assessed at $2 \mathrm{dpi}$ by measuring their average diameter on one leaf per plant, with a total of three plants assessed per sample. Two-way ANOVA showed significant effects of genotype $(P<0.01)$ and treatment $(P<0.01)$ and a significant interactive effect $(P<0.01)$; **, $P$ 
$<0.01$. c In planta fungal growth in leaves of each tested plant line. The growth rate of B. cinerea B1301 was measured as depicted above and indicated as BcActin/AtActin1. Two-way ANOVA indicated significant effects of genotype $(P<0.01)$ and treatment $(P<0.01)$ and a significant interactive effect $(P<$ $0.01) ; * *, P<0.01$. The data are presented as mean \pm SD from three biological replicates. The assays were repeated three times with similar results yielded.

\section{Supplementary Files}

This is a list of supplementary files associated with this preprint. Click to download.

- Additionalfile3FigureS3.tif

- Additionalfile2FigureS2.tif

- Additionalfile4Tables1.xls

- Additionalfile1Figures1.tif 